ARTICLE

Check for updates

httpst//doi.org/10.1038/s41467-021-22471-y

\title{
OPEN
}

\section{Ferroptotic cell death triggered by conjugated linolenic acids is mediated by ACSL1}

\author{
Alexander Beatty (1凶 ${ }^{1 凶}$, Tanu Singh ${ }^{1}$, Yulia Y. Tyurina ${ }^{2,3}$, Vladimir A. Tyurin ${ }^{2,3}$, Svetlana Samovich ${ }^{2,3}$, \\ Emmanuelle Nicolas' ${ }^{1}$, Kristen Maslar (10 ${ }^{4}$, Yan Zhou ${ }^{1}$, Kathy Q. Cai ${ }^{1}$, Yinfei Tan', Sebastian Doll ${ }^{5}$, \\ Marcus Conrad (1D 5,6, Aravind Subramanian7 , Hülya Bayır (1D 2,3,8, Valerian E. Kagan (1D 2,3,9,10,11,12, \\ Ulrike Rennefahrt ${ }^{13} \&$ Jeffrey R. Peterson (i) ${ }^{1 凶}$
}

Ferroptosis is associated with lipid hydroperoxides generated by the oxidation of polyunsaturated acyl chains. Lipid hydroperoxides are reduced by glutathione peroxidase 4 (GPX4) and GPX4 inhibitors induce ferroptosis. However, the therapeutic potential of triggering ferroptosis in cancer cells with polyunsaturated fatty acids is unknown. Here, we identify conjugated linoleates including $\alpha$-eleostearic acid ( $\alpha E S A$ ) as ferroptosis inducers. $\alpha$ ESA does not alter GPX4 activity but is incorporated into cellular lipids and promotes lipid peroxidation and cell death in diverse cancer cell types. $\alpha$ ESA-triggered death is mediated by acyl-CoA synthetase long-chain isoform 1 , which promotes $\alpha$ ESA incorporation into neutral lipids including triacylglycerols. Interfering with triacylglycerol biosynthesis suppresses ferroptosis triggered by $\alpha \mathrm{ESA}$ but not by GPX4 inhibition. Oral administration of tung oil, naturally rich in $\alpha E S A$, to mice limits tumor growth and metastasis with transcriptional changes consistent with ferroptosis. Overall, these findings illuminate a potential approach to ferroptosis, complementary to GPX4 inhibition.

\footnotetext{
${ }^{1}$ Fox Chase Cancer Center, Philadelphia, Pennsylvania, USA. ${ }^{2}$ Department of Environmental and Occupational Health, University of Pittsburgh, Pittsburgh PA, USA. ${ }^{3}$ Center for Free Radical and Antioxidant Health, University of Pittsburgh, Pittsburgh, PA, USA. ${ }^{4}$ Department of Biochemistry and Molecular Biology, Drexel University College of Medicine, Philadelphia, PA, USA. ${ }^{5}$ Institute of Metabolism and Cell Death, Helmholtz Zentrum München, Neuherberg, Germany. ${ }^{6}$ National Research Medical University, Laboratory of Experimental Oncology, Ostrovityanova 1, Moscow 117997, Russia. ${ }^{7}$ Broad Institute of Harvard and MIT, Cambridge, MA, USA. ${ }^{8}$ Department of Critical Care Medicine, Safar Center for Resuscitation Research, University of Pittsburgh, Pittsburgh, PA, USA. ${ }^{9}$ Department of Chemistry, University of Pittsburgh, Pittsburgh, PA, USA. ${ }^{10}$ Department of Pharmacology and Chemical Biology, University of Pittsburgh, Pittsburgh, PA, USA. ${ }^{11}$ Department of Radiation Oncology, University of Pittsburgh, Pittsburgh, PA, USA. ${ }^{12}$ Laboratory of Navigational Redox Lipidomics, IM Sechenov Moscow State Medical University, Moscow, Russia. ${ }^{13}$ Metanomics Health GmbH, Berlin, Germany.

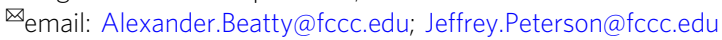




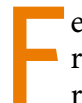
erroptosis is a form of non-apoptotic, iron-dependent, regulated cell death linked to lipid hydroperoxides, a form of reactive oxygen species (ROS) $)^{1-7}$. Interest in the therapeutic potential of inducing ferroptosis in cancer cells has led to a major research focus on inhibiting cellular antioxidant pathways that oppose ferroptosis. GPX4 is the only peroxidase known to efficiently reduce esterified, hydroperoxy fatty acids into unreactive alcohols ${ }^{8}$. Recently, ferroptosis suppressor protein 1 (FSP1) was identified as a second ferroptosis suppression mechanism through its recycling of coenzyme $\mathrm{Q}_{10}$, a radical-trapping antioxidant ${ }^{9,10}$. Small molecules that trigger ferroptosis identified thus far include agents that inhibit GPX4 directly, molecules that deplete the GPX4 cofactor glutathione, and compounds that oxidize iron $1,9,11,12$. Lipid hydroperoxides are generated by nonenzymatic oxidation or by lipoxygenases or cytochrome P450 oxidoreductase $^{13-15}$. Hydroperoxides disrupt membrane architecture, produce reactive aldehydes, and drive cell death ${ }^{16}$. In contrast to therapeutic targeting of antioxidant pathways, we have pursued a complementary strategy for ferroptosis induction based on enhancing the production of lipid hydroperoxides.

Hydroperoxides can spread by a free radical-mediated chain reaction leading to oxidation of adjacent polyunsaturated acyl chains, and consequently higher levels of this species increase vulnerability to peroxide propagation. Indeed, supplementing cells with arachidonic acid sensitizes them to ferroptosis triggered by GPX4 inhibitors ${ }^{17,18}$. Similarly, clear-cell carcinomas are rendered vulnerable to GPX4 inhibitors due to HIF-2a-dependent polyunsaturated lipid accumulation ${ }^{19}$. Conversely, increasing the relative proportion of monounsaturated acyl chains at the expense of polyunsaturated lipids suppresses sensitivity to ferroptosis ${ }^{20,21}$. Thus, increasing polyunsaturated lipids in cancer cells could enhance their vulnerability to ferroptosis. Importantly, this approach could exploit the propensity of cancer cells to scavenge fatty acids from their environment ${ }^{22}$.

Here, we report that linolenic fatty acids with conjugated double bonds, produced by certain plant species, can induce ferroptosis in diverse cancer cells as a single agent. Conjugated linoleate triggers ferroptosis by a mechanism distinct from canonical ferroptosis inducers. Cell death is mediated by acylCoA synthetase long-chain family member 1 (ACSL1), implicating this isoform in ferroptosis. Furthermore, oral administration of tung tree seed oil, naturally rich in the conjugated linoleate $\alpha$-eleostearic acid ( $\alpha \mathrm{ESA})$, inhibits tumorigenesis and metastasis in a murine breast cancer xenograft model. aESA metabolites are detected in tumors of treated mice and are associated with the expression of a ferroptotic gene signature. These results introduce a distinct class of ferroptosis inducers and offer insights into the molecular basis of ferroptotic sensitivity. The tractability of these dietary, pro-ferroptotic fatty acids addresses the current lack of effective GPX4 inhibitors for use in vivo and suggests an opportunity to exploit a metabolic liability across cancer subtypes.

\section{Results}

Glutathione depletion triggers ferroptosis in triple-negative breast cancer cells. Several triple-negative breast cancer cell lines are killed by the glutathione biosynthesis inhibitor buthionine sulfoximine (BSO) (Fig. 1a and ref. ${ }^{23}$ ). However, the specific cellular pool of ROS responsible for cell death is unclear. Glutathione depletion in some contexts induces ferroptosis ${ }^{24}$, which is operationally defined by three criteria; its association with lipid peroxidation, the ability to suppress cell death with lipophilic antioxidants like ferrostatin-1 (fer-1), and iron-dependence ${ }^{1}$. Consistent with ferroptosis, cytotoxicity in BSO-treated BT-549 cells was blocked by fer-1 but not by Z-VAD-FMK, an inhibitor of apoptosis (Fig. 1a, b). Similarly, iron chelators deferoxamine and deferiprone suppressed BSO-mediated cell death (Fig. 1c, Supplementary Fig. 1a). Furthermore, BSO treatment resulted in the accumulation of lipid peroxidation products and this was suppressed by fer-1 (Fig. 1d, e). Thus, glutathione depletion triggers death consistent with ferroptosis in BT-549 cells and identifies lipid hydroperoxides as the lethal ROS species underlying glutathione addiction in these cells.

Sensitivity to ferroptosis is associated with the accumulation of polyunsaturated lipids. Fer-1 suppressed BSO toxicity in half of the triple-negative breast cancer (TNBC) cell lines tested, consistent with ferroptosis (Fig. 1f, red bars). These cell lines were also generally more sensitive to ML162, a small molecule inhibitor of GPX4 ${ }^{25}$ (Welch's t-test, $p=0.01$, Fig. 1g, Supplementary Fig. 1b). Thus, fer-1-suppressible cell death is a common response to glutathione depletion in a subset of TNBC cells and is associated with a greater dependence on GPX4 activity. This vulnerability was not due to decreased GPX4 expression (Supplementary Fig. 1c). We used ANOVA of our prior metabolomic data for these cell lines ${ }^{23}$ to compare levels of individual metabolites between TNBC cell lines in which fer-1 rescued BSOmediated cell death and those in which it did not (Fig. 1f), and found that two of the three most significantly differentially accumulated metabolites were linoleate (18:2)-substituted phosphatidylcholines (Fig. 1h, i). These membrane phospholipids were more enriched in cell lines that undergo fer-1-suppressible death. This finding suggested the hypothesis that polyunsaturated lipid enrichment may increase dependence on GPX4 and vulnerability to ferroptosis (Fig. 1g).

aESA triggers cancer-selective ferroptosis. We postulated that elevating polyunsaturated lipid levels might sensitize TNBC cells to ferroptosis. We tested a variety of fatty acids and identified $\alpha$-eleostearic acid [(9Z,11E,13E)-octadeca-9,11,13-trienoic acid; $\alpha E S A$ ] (Fig. 2a) as a fatty acid that enhanced cell death in combination with BSO (Fig. 2b). aESA is abundant in certain plants (e.g. bitter melon and tung tree) ${ }^{26}$ and was previously shown to suppress the growth of breast cancer cells in a manner reversible by antioxidants ${ }^{27}$. Others have suggested that aESA triggers apoptotic cell death ${ }^{28-33}$ although a role for ferroptosis was not investigated. Unexpectedly, we found that aESA triggered cell death as a single agent and this death was suppressed by fer- 1 (Fig. 2b), the iron chelator deferoxamine (Fig. 2c), and by the lipophilic antioxidant vitamin E (Supplementary Fig. 2a), and was associated with an increase in lipid peroxidation products that could be suppressed by fer-1 (Fig. 2d). Neither Z-VAD-FMK nor the necroptosis inhibitor nec-1s blocked aESA-induced cell death (Supplementary Fig. 2b, c). Together, these findings show that aESA induces ferroptosis as a single agent and distinguishes aESA from arachidonic and adrenic fatty acids, which sensitize cells to GPX4 inhibition but do not induce ferroptosis ${ }^{13,17}$.

The non-transformed MCF-10A (Fig. 2e) and MCF-12A (Supplementary Fig. 2d) breast epithelial cell lines were resistant to death by aESA. By contrast, all TNBC cell lines were susceptible and, in all cases, death was prevented by fer-1 (Fig. 2b, f). Notably, even cell lines that did not undergo ferroptosis in response to glutathione depletion (Fig. 1f) were sensitive to aESA, pointing to a distinct mechanism of ferroptosis induction. Timelapse microscopy of aESA-treated BT549 cells (Supplementary Movie 1) showed that during the latter part of $24 \mathrm{~h}$ of treatment, otherwise healthy appearing cells undergo a sudden death, morphologically similar to cell death by the canonical ferroptosis inducer ML162 (Supplementary Movie 2) and distinct from apoptosis (Supplementary Movie 3). 
a

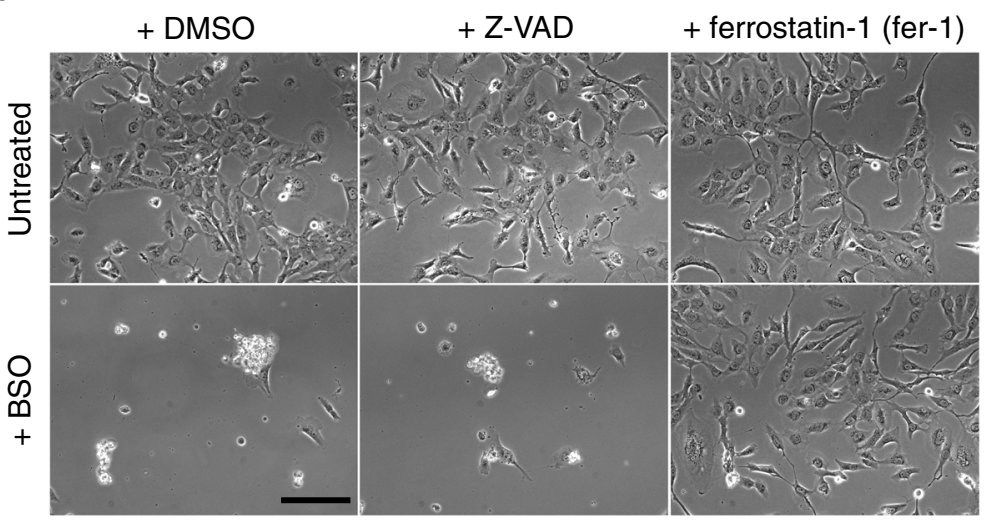

BT-549 (TNBC cell line)

d

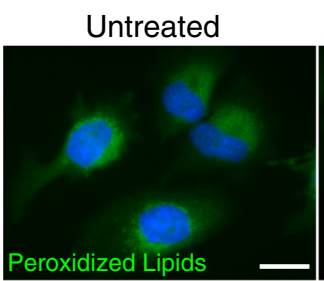

BT-549
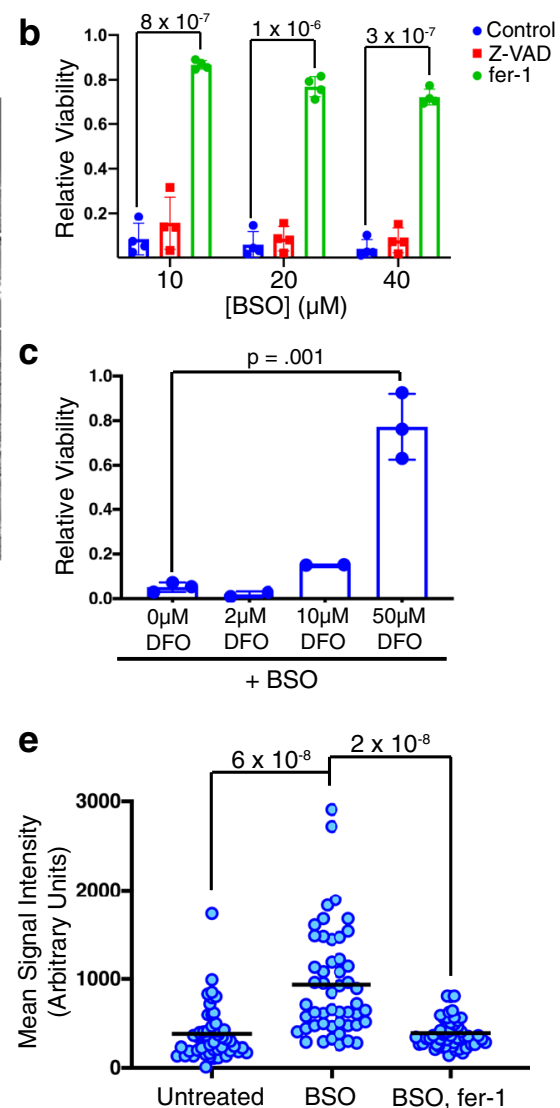

f

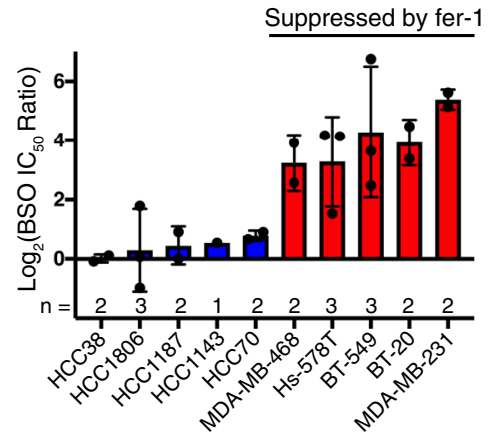

h

Phosphatidylcholine (C18:0, C18:2)

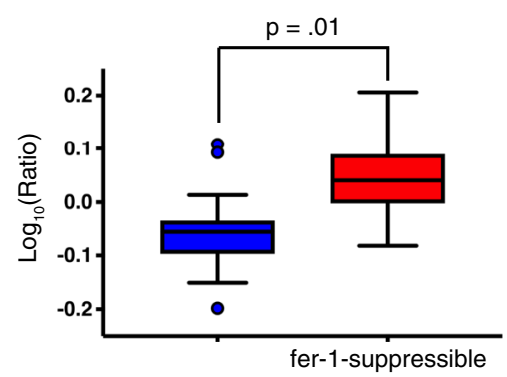

g

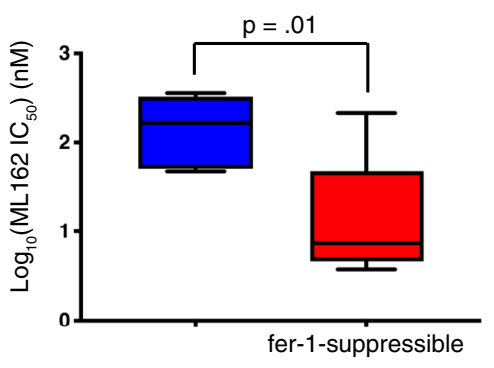

i

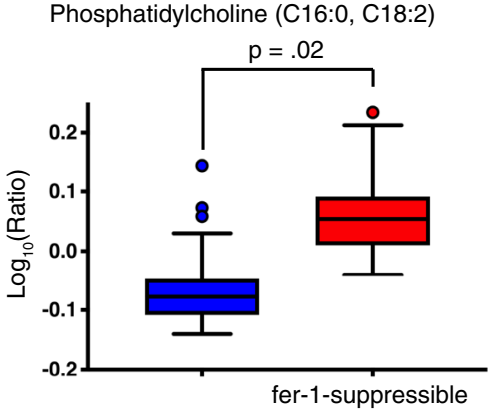

aESA is incorporated into diverse cellular lipids. To examine how $\alpha E S A$ is metabolized, we conducted a lipidomic analysis of MDA-MB-231 cells treated with aESA for three hours, prior to overt cell death. Lipids containing acyl chains with 18 carbons and three double bonds (18:3), consistent with $\alpha \mathrm{ESA}$, were rare in untreated cells (Fig. 2g, blue bars), but were found in diverse lipids in aESA-treated cells, including phospholipids and neutral lipids (green bars; Supplementary Data 1). Fer-1 co-treatment did not dramatically alter the spectrum of lipid classes that incorporated 18:3 acyl chains (compare green \& purple bars). The relative abundance of di- and triacylglycerol lipids increased $\sim 2$ fold in aESA and aESA + fer-1-treated cells compared to untreated controls, consistent with aESA being incorporated into storage lipids (Fig. 2h). aESA-induced lipidomic changes were not downstream consequences of ferroptosis because they were not replicated by ML162 treatment (orange bars in Fig. 2g, h). 
Fig. 1 Glutathione depletion triggers ferroptosis in a subset of TNBC cell lines and elevated polyunsaturated fatty acid levels are associated with vulnerability to ferroptosis. a Light micrographs of BT-549 cells treated with $10 \mu \mathrm{M}$ BSO for $72 \mathrm{~h}$ in the presence or absence of $20 \mu \mathrm{M} Z$-VAD-FMK or fer1. Scale bar represents $200 \mu \mathrm{m}$. b BT-549 relative cell viability after $72 \mathrm{~h}$ of BSO treatment with vehicle, $20 \mu \mathrm{M} Z-\mathrm{VAD}-\mathrm{FMK}$, or $2 \mu \mathrm{M}$ fer- $1(n=4$ independent experiments). Error bars, here and below, denote standard deviation centered on the mean. The numbers above the brackets are $\mathrm{p}$ values from Student's t-tests (two-sided) unless otherwise noted. p values were not corrected for multiple testing unless stated. c Relative viability of BT-549 cells treated with $10 \mu \mathrm{M}$ BSO and the indicated concentration of deferoxamine (DFO) for $72 \mathrm{~h}(n=3)$. Values were normalized to account for loss of viability associated with DFO. d Representative fluorescent micrographs of BT-549 cells treated with vehicle, BSO ( $50 \mu \mathrm{M})$, or BSO and fer- 1 ( $2 \mu \mathrm{M})$ for $48 \mathrm{~h}$. Green corresponds to cellular macromolecules modified with peroxidized lipid breakdown products (Click-iT lipid peroxidation detection kit, ThermoFisher Scientific). DNA is stained blue. Scale bar represents $20 \mu \mathrm{m}$. e Quantitation of lipid peroxidation products from individual cells ( $n=50$ per condition) in d. Lines represent the mean. $\mathbf{f}$ The $\log _{2}$-transformed ratio of the $I_{50}$ of BSO in the presence or absence of $2 \mu \mathrm{M}$ fer- 1 for each cell line. Cell lines in which fer-1 reduced cell death from BSO > 8-fold are designated "fer-1-suppressible". $\mathbf{g}$ Box and whiskers plot of the $\log _{2}$-transformed IC 50 values for ML162 in non-fer-1-suppressible (blue) and fer-1-suppressible (red) TNBC cell lines (as defined in $\mathbf{f}$ ). The line represents the median value, the box defines the interquartile range (25th to 75th percentile), and the whiskers show minimum and maximum values. $\mathbf{h}$ Box plots showing the log 10 -transformed, mediannormalized relative levels of phosphatidylcholine (C18:0, C18:2) and i phosphatidylcholine (C16:0, C18:2) in non-fer-1-suppressible and fer-1-suppressible TNBC cell lines. Each cell line is represented by at least 5 replicates. $p$ values based on ANOVA and corrected for multiple testing. The line shows the median value, the box shows the interquartile range, the whiskers represent the upper and lower adjacent values, and outliers are shown as dots. Source data are provided as a Source Data file.

Structure-activity analysis of conjugated PUFAs. To characterize structural features of aESA required for ferroptosis, we screened related conjugated and unconjugated polyunsaturated fatty acids for death induction. aESA possesses conjugated double bonds at carbons 9, 11, and 13 with cis, trans, trans stereochemistry, respectively (Fig. 2a). We examined cis-trans stereoisomers of $\alpha \mathrm{ESA}$ and found that isomerization at position 9 ( $\beta$-eleostearic acid) or at both 9 and 13 (catalpic acid) retained cell death activity while isomerization at position 13 alone (punicic acid) led to a $\sim 4$-fold reduction in potency (Fig. 3a). Jacaric acid (conjugated 18:3, cis-8, trans-10, cis-12) showed the most potent cell killing $\left(1.8 \mu \mathrm{M} \mathrm{IC}_{50}\right)$, demonstrating that shifting double bond positioning while maintaining the sequential cis, trans, cis stereochemistry of punicic acid, did not disrupt activity. $a-$ calendic acid (conjugated 18:3, trans-8, trans-10, cis-12), a stereoisomer of jacaric acid, was similarly potent. Thus, cell killing is not strictly dependent on the precise positioning or stereochemistry of double bonds within the aliphatic chain. Conjugated linoleic acid (18:2), which shares similar position and stereochemistry of its two double bonds with aESA, was a poor inducer of cell death, however, suggesting that the third conjugated double bond is required for activity. Furthermore, all tested non-conjugated polyunsaturated fatty acids (PUFAs) were much less potent single-agent inducers of cell death (Supplementary Fig. 2e) including arachidonate, which enhances ferroptosis in some contexts ${ }^{17,18}$. Cell killing by arachidonate could not be rescued by fer-1, liproxstatin or deferoxamine, highlighting an important distinction between the activity of conjugated and non-conjugated PUFAs (Supplementary Fig. 2f). By contrast, jacaric- and catalpic acid-induced death was suppressed by fer- 1 (Supplementary Fig. 2g). Similar results were found in BT-549 cells (Supplementary Fig. 2h), demonstrating the conserved ability of conjugated 18:3 fatty acids to trigger ferroptosis.

To validate the relative potency of these fatty acids as inducers of cell death, we profiled their effects on viability across 100 human cancer cell lines at 6 doses using the PRISM screening platform ${ }^{34}$. Thunor ${ }^{35}$ was used to fit dose-response curves and the area over the curve (Activity Area) was calculated to reflect the potency of cell killing ${ }^{35}$. A histogram reflecting the range of Activity Areas across the cell line panel for each fatty acid is presented as a violin plot in Fig. 3b. Jacaric and catalpic acid were consistently most toxic to the panel, followed by $\alpha$-calendic acid, $\alpha E S A$ and $\beta E S A$. Whether the differential potency of these conjugated linolenic acids is related to their metabolism by specific enzymes or due to differences in solubility or other physicochemical properties is an important open question.
Punicic acid and arachidonic acid exhibited substantially less anti-proliferative activity and the remaining control fatty acids were largely inactive. These results support a unique deathinducing activity of conjugated linolenic acids.

The tissue of origin of cell lines did not appear to correlate with aESA sensitivity or resistance. However, the sensitivity of individual cell lines to one conjugated linolenate was correlated with sensitivity to the others, but not with control fatty acids (Fig. 3c). Taken together these results show that conjugated linolenic acids exhibit broad toxicity against multiple cancer cell lines of distinct tissue origin. Furthermore, sensitivity or resistance to conjugated linolenic acids is intrinsic to each cell line.

The role of GPX4 in aESA-induced ferroptosis. Next, we sought mechanistic insights into ferroptosis induction by aESA. One possibility is that aESA triggers ferroptosis by inhibiting GPX4 directly or indirectly by depleting glutathione. Unlike BSO, treatment with aESA did not significantly affect total cellular glutathione levels despite decreasing cell viability (Fig. 4a). Direct GPX4 inhibitors have been shown to reduce GPX4 protein levels ${ }^{17}$. We confirmed this for ML162 and RSL3 but found that aESA or the negative control erastin had no effect on GPX4 levels (Fig. 4b), suggesting that aESA does not directly inhibit GPX4. As an alternative approach to test if aESA inhibits GPX4, we compared the kinetics of ferroptosis in MDA-MB-231 cells treated with aESA, ML162, and RSL3 alone and in combination. Combining both GPX4 inhibitors at $500 \mathrm{nM}$ triggered fer-1suppressible cell death with similar kinetics to either GPX4 inhibitor alone, indicating this was a saturating dose of inhibitor (Fig. 4c). In contrast, cell viability was lost significantly more rapidly when cells were co-treated with $500 \mathrm{nM} \mathrm{ML162}$ and $50 \mu \mathrm{M}$ aESA, a dose of $\alpha \mathrm{ESA}$ that took longer to induce cell death than either of the GPX4 inhibitors alone. Consistent with ferroptosis, cell death could be rescued by fer-1 (green in Fig. 4c). The ability of aESA to enhance the kinetics of cell death when GPX4 is maximally inhibited shows that aESA and GPX4 inhibitors trigger ferroptosis by distinct mechanisms.

aESA enhanced the toxicity of GPX4 inhibitors in multiple cell lines (Fig. 4d and Supplementary Fig. 3), suggesting that the reduction of hydroperoxides by GPX4 opposes aESA-induced ferroptosis. Consistent with this model, transient knockdown of GPX4 by siRNA also enhanced cell killing by aESA (Fig. 4e). Finally, we generated MDA-MB-231 and BT549-derived cell lines stably overexpressing GPX4 or eGFP as control (Fig. 4f) and found that cell lines overexpressing GPX4 were resistant to 
a<smiles>CCCC/C=C/C=C\C=C/CCCCCCC(=O)O</smiles>

b

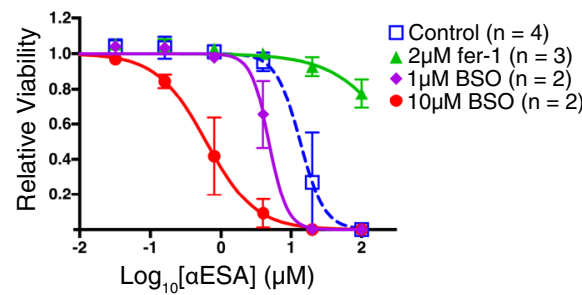

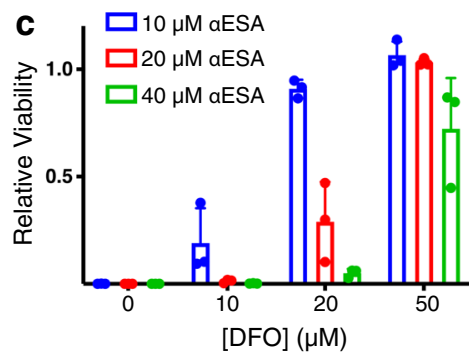

f

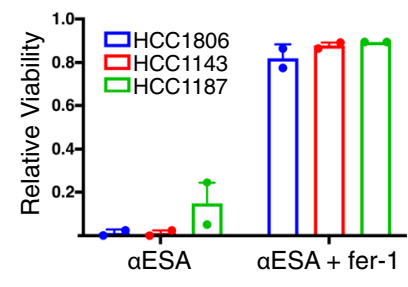

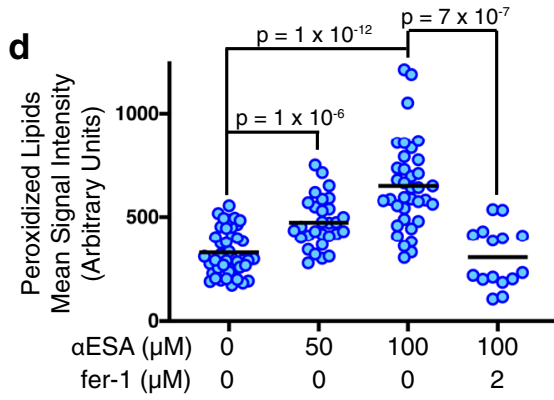

e

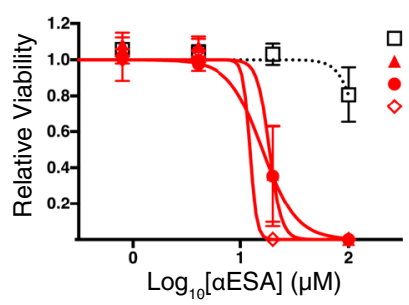

$\square$ MCF-10A $(n=3)$

BT-549 $(\mathrm{n}=3)$

MDA-MB-231 $(n=3)$

MDA-MB-468 $(n=2)$

g

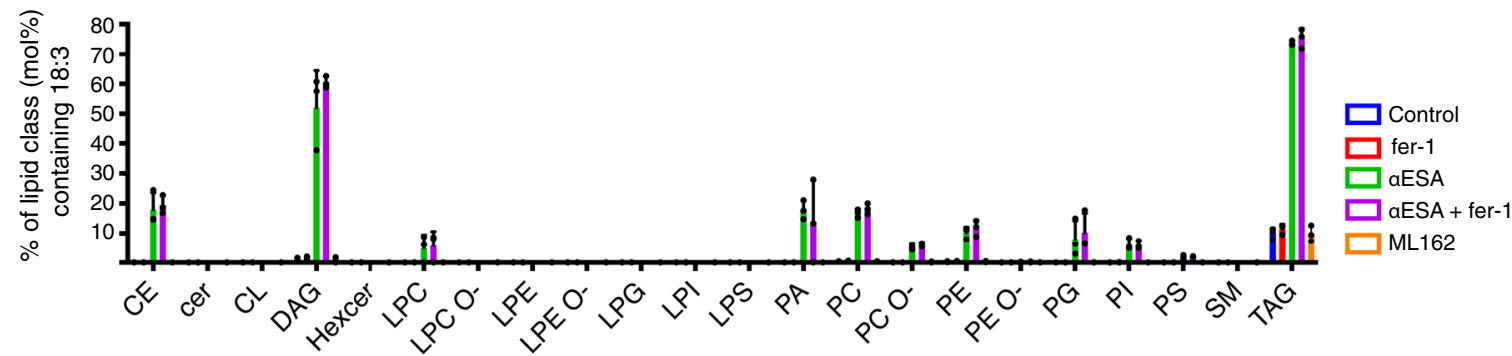

h

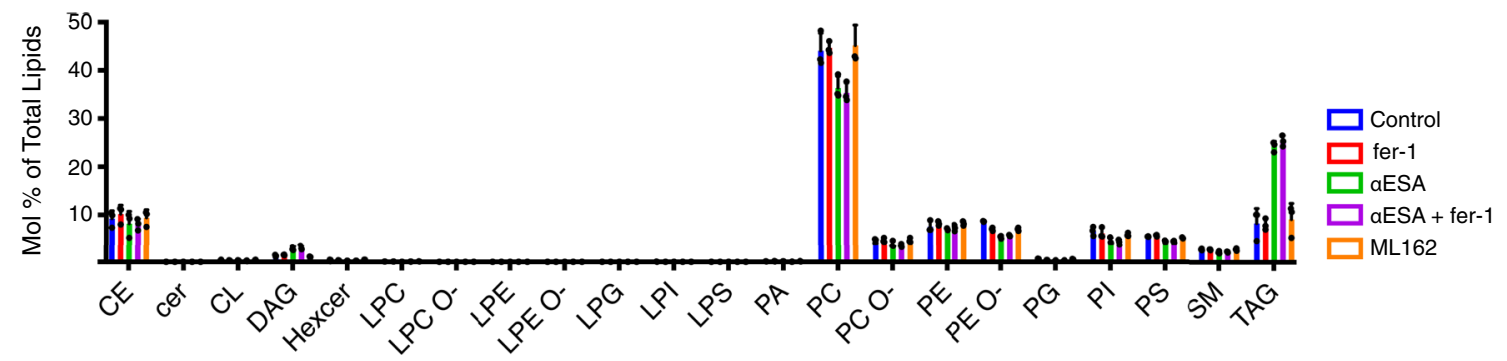

Fig. 2 The conjugated linolenic fatty acid $\alpha$-eleostearic acid is incorporated into cellular lipids and induces ferroptosis. a Structure of $\alpha$-eleostearic acid $(\alpha E S A)$. b Cell viability dose-response curves for $\alpha$ ESA and the specified additional compound in MDA-MB-231 cells. Cells were treated for $72 \mathrm{~h}$. Error bars in this and subsequent panels represent standard deviation centered on the mean. $\mathbf{c}$ Relative viability of MDA-MB-231 cells incubated with the indicated doses of $\alpha$ ESA and DFO for $72 \mathrm{~h}$ ( $n=3$ independent experiments). Values were normalized to account for loss of viability associated with DFO. d Quantitation of lipid peroxidation products in individual cells after $4 \mathrm{~h}$ of treatment with the specified agent. The line indicates the mean. From left to right, $n=42,29,36$, and 15. $p$ values from two-sided Student's $t$ tests are shown. e Cell viability dose-response curves for $\alpha$ ESA for three TNBC cell lines (red lines) and noncancerous MCF-10A controls (black dashed line) after $72 \mathrm{~h}$ of treatment. $\mathbf{f}$ Relative cell viability for three TNBC cell lines after $72 \mathrm{~h}$ of treatment with $\alpha \mathrm{ESA}$ or the combination of $\alpha \mathrm{ESA}$ and fer- $1(2 \mu \mathrm{M})(n=2$ independent experiments). The dose $\alpha \mathrm{ESA}$ was $100 \mu \mathrm{M}$ for HCC1806 and HCC1143 and $20 \mu \mathrm{M}$ for HCC1187. $\mathbf{g}$ Percent of the mole fraction for each of the indicated classes of lipid that contain 18:3 (18 carbon, 3 double bonds) fatty acids consistent with $\alpha$ ESA ( $n=3$ biological replicates for each condition). $\mathbf{h}$ Mole percent of each lipid class as a fraction of total lipids in MDA-MB-231 cells incubated with vehicle, $2 \mu \mathrm{M}$ fer-1, $50 \mu \mathrm{M} \alpha \mathrm{ESA}, 50 \mu \mathrm{M} \alpha \mathrm{ESA}$ and $2 \mu \mathrm{M}$ fer-1, or $250 \mathrm{nM}$ ML162 for $3 \mathrm{~h}(n=3$ biological replicates for each condition). CE $=$ cholesterol esters, Cer = ceramide, DAG = diacylglycerol, Hexcer = hexosylceramide, LPC = lysophosphatidylcholine, LPC O- = ether-linked lysophosphatidylcholine, LPE = lysophosphatidylethanolamine, LPE O- = ether-linked lysophosphatidylethanolamine, LPG = lysophosphatidylglycerol, LPI = lysophosphatidylinositol, LPS = lysophosphatidylserine, PA = phosphatidate, PC = phosphatidylcholine, $\mathrm{PC} O$ - = ether-linked phosphatidylcholine, $\mathrm{PE}=$ phosphatidylethanolamine, PE O- = ether-linked phosphatidylethanolamine, $\mathrm{PG}=$ phosphatidylglycerol, $\mathrm{PI}=$ phosphatidylinositol, $\mathrm{PS}=$ phosphatidylserine, $\mathrm{SM}=\mathrm{sphingomyelin}$, TAG = triacylglycerol. Source data are provided as a Source Data file.

ML162-triggered ferroptosis (Fig. 4g) and aESA-induced ferroptosis, though the resistance to ferroptosis triggered by aESA was less robust in BT-549 cells (Fig. 4h).

FSP1 mediates a second pathway for hydroperoxide neutralization that operates in parallel to GPX4. By reducing coenzyme $\mathrm{Q}_{10}$, FSP1 maintains an active pool of this radical-trapping lipophilic antioxidant ${ }^{9,10}$. As expected, iFSP1, a small-molecule inhibitor of FSP $1^{9}$, enhanced cell killing by ML162 in both BT-549 and MDA-MB-231 cells (Fig. 4i). By contrast, iFSP1 had no effect on aESA toxicity in BT-549 cells, but enhanced cell death in MDAMB-231 cells (Fig. 4j), highlighting a further distinction between ferroptosis induced by aESA and canonical ferroptosis inducers. 
a

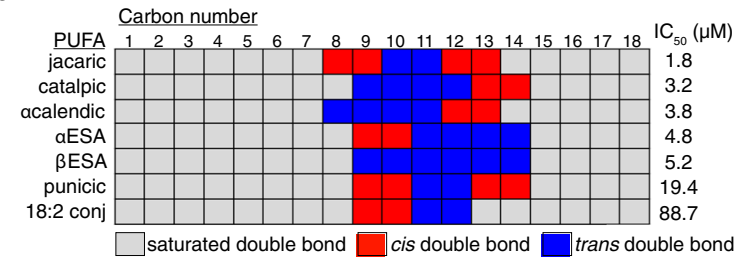

c

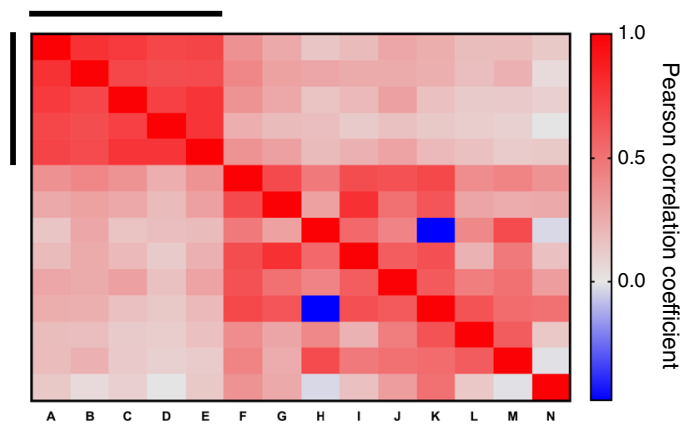

b

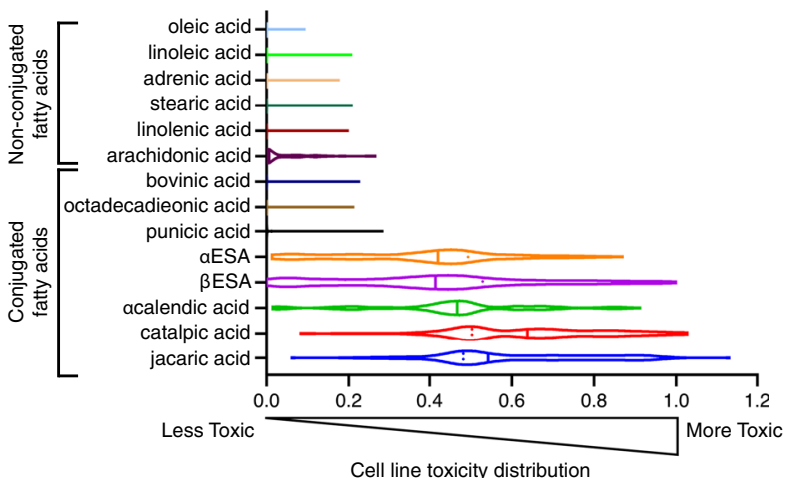

Cell line toxicity distribution

Fig. 3 Conjugated linolenic fatty acids are generally toxic to diverse cancer cells. a Schematic representing the location and double bond geometry of 18 carbon conjugated polyunsaturated fatty acids. Cell viability $I C_{50}$ values are shown for each conjugated PUFA from at least two biological replicate measurements in MDA-MB-231 cells after $72 \mathrm{~h}$ of treatment. $\mathbf{b}$ Assessment of fatty acid toxicity across cancer cell lines. A mixture of 100 barcoded human cancer cell lines were treated in triplicate with the indicated fatty acid at doses from 0-35 $\mu \mathrm{M}$. Viable cells of each cell line in the mixture were quantified at $48 \mathrm{~h}$ of treatment. A violin plot illustrates the distribution of cell line-specific toxicities (calculated as the area over the dose dependent-cell viability curve across the treatment dose range). A toxicity value of 1 denotes complete loss of viable cells and 0 corresponds to viability similar to vehicle-treated controls. Dark bars denote median toxicity against all cell lines in the panel and dotted lines indicate quartile boundaries. $\mathbf{c}$ Heatmap presenting the Pearson correlation coefficients of the activity areas across the cancer cell line panel in $\mathbf{b}$ for each pair of fatty acids in the test set of fourteen. Red shows positive correlation and blue indicates negative correlation. The bars denote the position of the following conjugated linolenic acids from left to right and top to bottom: jacaric acid, catalpic acid, $\alpha$-calendic acid, $\beta E S A$, and $\alpha E S A$. Source data are provided as a Source Data file.

aESA-induced ferroptosis is ACSL1-dependent. The first step in esterification of fatty acids into lipids is their conjugation to coenzyme A (CoA), catalyzed by acyl-CoA fatty ligases. There are five long chain-specific isoforms (ACSLs) that are distinguished by fatty acid specificity and subcellular localization ${ }^{36}$. ACSL4 preferentially esterifies certain PUFAs including arachidonic acid and loss of ACSL4 protects cells from ferroptosis induced by direct or indirect inhibition of GPX4 (Fig. 5a) ${ }^{37-39}$. aESA, however, was similarly toxic to ACSL4-deficient Pfal cells and control parental cells (Fig. 5b), supporting a difference in the mechanism of action of aESA and canonical ferroptosis inducers. aESAinduced cell death was rescued by fer- 1 in both cell lines, consistent with ferroptosis (Supplementary Fig. 4a). To identify ACSL isoforms that contribute to aESA-induced ferroptosis, we individually knocked down expression of each of the five isoforms in BT-549 and MDA-MB-231 cells by RNAi (Supplementary Fig. 4b, c). As expected, ML162 toxicity was suppressed by ACSL4 depletion in both cell lines (Fig. $5 c$ and Supplementary Fig. $4 d-f$ ). aESA toxicity, however, was significantly suppressed by knockdown of ACSL1, though other ACSL isoforms may also contribute.

We used CRISPR/Cas9 to generate multiple clonal BT-549 cell lines deficient in ACSL1 (Fig. 5d). ACSL1 loss reduced aESA sensitivity compared to controls (Fig. 5e) and increased sensitivity to ML162 (Fig. 5f). Similar results were obtained using an independent guide RNA (Supplementary Fig. 4g, h). Reexpressing CRISPR-resistant ACSL1 in ACSL1-deficient cells restored aESA sensitivity (Fig. 5g), and increasing ACSL1 expression in control BT-549 cells further enhanced aESA toxicity (Fig. 5h). These results demonstrate that ACSL1 promotes aESA-triggered cell death. Furthermore, they highlight a mechanistic distinction between $\alpha E S A$ and canonical ferroptosis inducers and expand the spectrum of ACSL isoforms involved in ferroptosis.

ACSL1 regulates aESA metabolism. To elucidate the impact of ACSL1 on lipid metabolism, we conducted a lipidomic analysis of two independent ACSL1-deficient cell lines as well as an ACSL1overexpressing line and their parental control cells (Fig. 6). Overall, the pattern of lipid enrichment or depletion in response to ACSL1 modulation was generally similar in the absence or presence of aESA (compare Fig. 6 left and middle panels). Among the differentially expressed lipids, we noted that diacylglycerols (DAGs) and triacylglycerols (TAGs) were significantly reduced in ACSL1-deficient cells compared to controls or ACSL1-overexpressing cells treated with aESA (Supplementary Data 2). These findings are consistent with prior studies linking ACSL1 to the production of these lipids ${ }^{40,41}$.

Next, we examined the impact of ACSL1 specifically on lipids bearing 18:3 acyl chains (Fig. 6, right). The mole fraction of 18:3containing lipids as a class was modestly but significantly decreased in aESA-treated $A C S L 1^{-1-}$ cells compared to aESAtreated controls ( $p=0.006$, Fisher's combined test). This suggests that the resistance of ACSL1-deficient cells to aESA could be related to decreased aESA incorporation. Lipid subgroup-focused analysis revealed 18:3 decreases specifically in phosphatidylglycerols, DAGs, and TAGs (Supplementary Data 3). Conversely, we observed a significant increase in 18:3-bearing cholesterol esters and vinyl ether-linked phosphatidylcholine and phosphatidylethanolamine in aESA-treated ACSL1-deficient cells. A potential explanation is that aESA may also be weakly utilized by other ACSL isoforms such as ACSL4, which is known to direct substrates into cholesterol esters ${ }^{40}$. Similar and more robust 

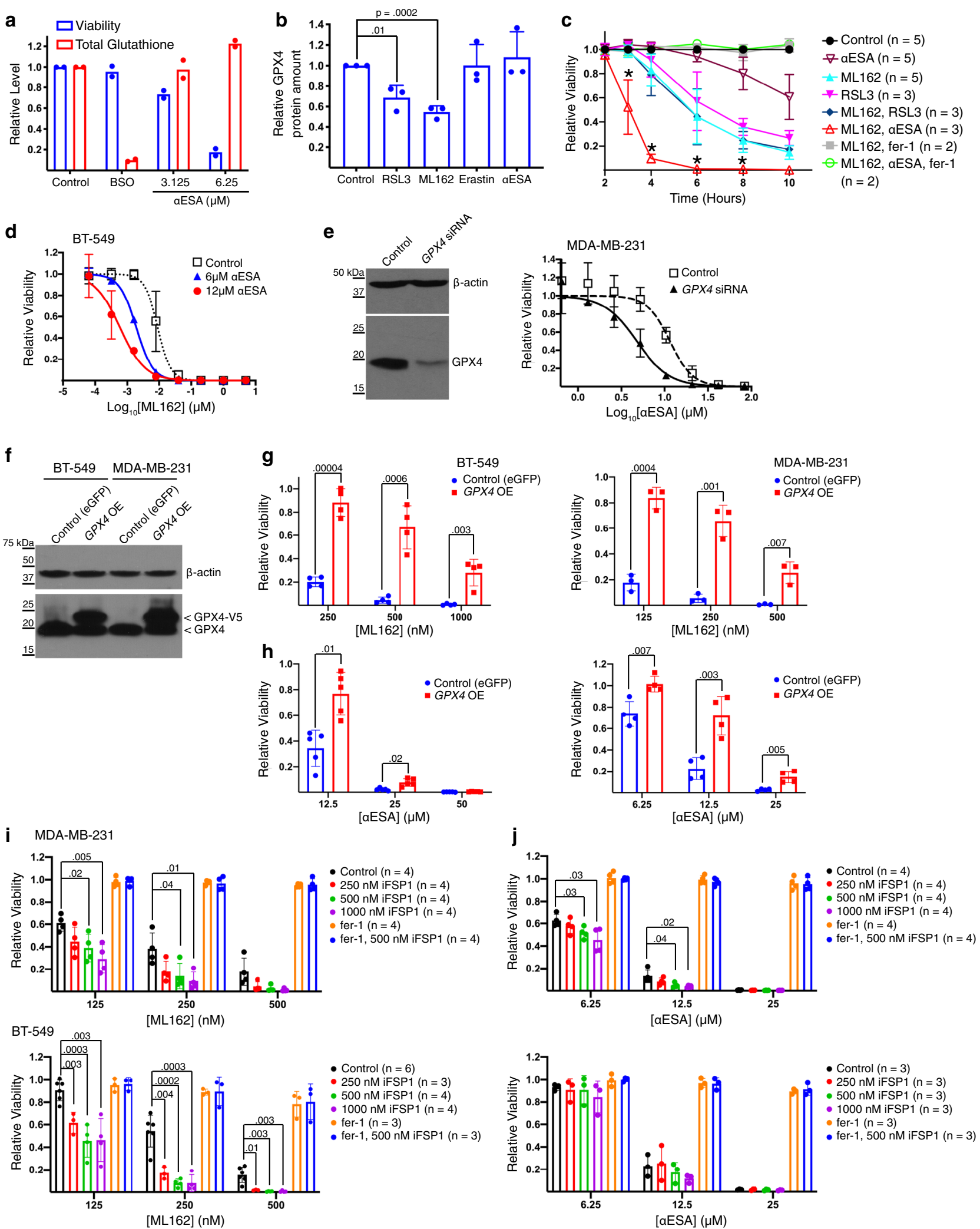

differences were observed comparing ACSL1-deficient to ACSL1overexpressing cells. They included significantly lower 18:3bearing lipids overall $(p=0.0002$, Fisher's combined test $)$ and particularly in DAGs $\left(p=1 \times 10^{-10}\right)$ and TAGs $(p=0.0002)$. In an independent experiment, we confirmed that transient silencing of ACSL1 expression (Fig. 7a) reduced 18:3 chain incorporation into TAG species of ESA-treated cells (Fig. 7b). Together these findings demonstrate a role for ACSL1 in the incorporation of aESA and other fatty acids into DAGs/TAGs, which could suggest a role for lipid droplets in aESA-induced ferroptosis.

aESA triggers ACSL1-dependent increases in lipid hydroperoxides. We next sought to characterize aESA-induced lipid 
Fig. 4 Mechanistic analysis of $\boldsymbol{\alpha E S A}$-induced ferroptosis. a Relative viability (blue) and levels of total glutathione (red) in MDA-MB-231 cells after $24 \mathrm{~h}$ of treatment with $20 \mu \mathrm{M} \mathrm{BSO}$, or the specified dose of $\alpha \mathrm{ESA}(n=2$ independent experiments). b Densitometric quantification of GPX4 protein levels from western blots of lysates from MDA-MB-231 cells that were treated for $4 \mathrm{~h}$ with the indicated agent. $n=3$ independent experiments. Error bars in this and subsequent panels represent standard deviation centered on the mean. $p$ values in this and subsequent panels were calculated using two-sided Student's $t$ tests. c Cell viability over time for MDA-MB-231 cells treated as specified. * denote $p$ values comparing viability of cells treated with ML162 and RSL3 to cells treated with $\alpha E S A$ and ML162. $p$ values from left to right are $0.003,0.0001,0.02$, and 0.008 . $\mathbf{d}$ Cell viability dose-response curves for ML162 and the indicated dose of $\alpha$ ESA in BT-549 cells after $72 \mathrm{~h}$ of treatment ( $n=2$ independent experiments). e Western blot showing GPX4 protein level in MDA-MB231 cells $48 \mathrm{~h}$ after transfection with a pool of GPX4-targeted siRNA or non-targeting siRNA. $\beta$-actin is the loading control for this and the subsequent western blots unless otherwise specified. The right panel shows cell viability dose-response curves for $\alpha$ ESA in GPX4-depleted and control MDA-MB-231 cells ( $n=3$ independent experiments). $\alpha$ ESA was added to cells $48 \mathrm{~h}$ after transfection for $24 \mathrm{~h}$. $\mathbf{f}$ Western blot of endogenous GPX4 and exogenously expressed V5-tagged GXP4 in BT-549 and MDA-MB-231 cells. Relative viability of these cells after $48 \mathrm{~h}$ incubation with the indicated dose of $\mathbf{g}$, ML162 ( $n=4$ and 3 for BT-549 and MDA-MB-231, respectively) or $\mathbf{h}, \alpha$ ESA ( $n=5$ and 4 for BT-549 and MDA-MB-231, respectively). $p$-values from Student's t-tests are shown. Relative viability of MDA-MB-231 or BT-549 cells after $48 \mathrm{~h}$ of treatment with the indicated dose of either $\mathbf{i}, \mathrm{ML} 162$ or $\mathbf{j}, \alpha \mathrm{ESA}$ and vehicle, iFSP1, fer-1 $(2 \mu \mathrm{M})$, or iFSP1 and fer-1. Source data are provided as a Source Data file.
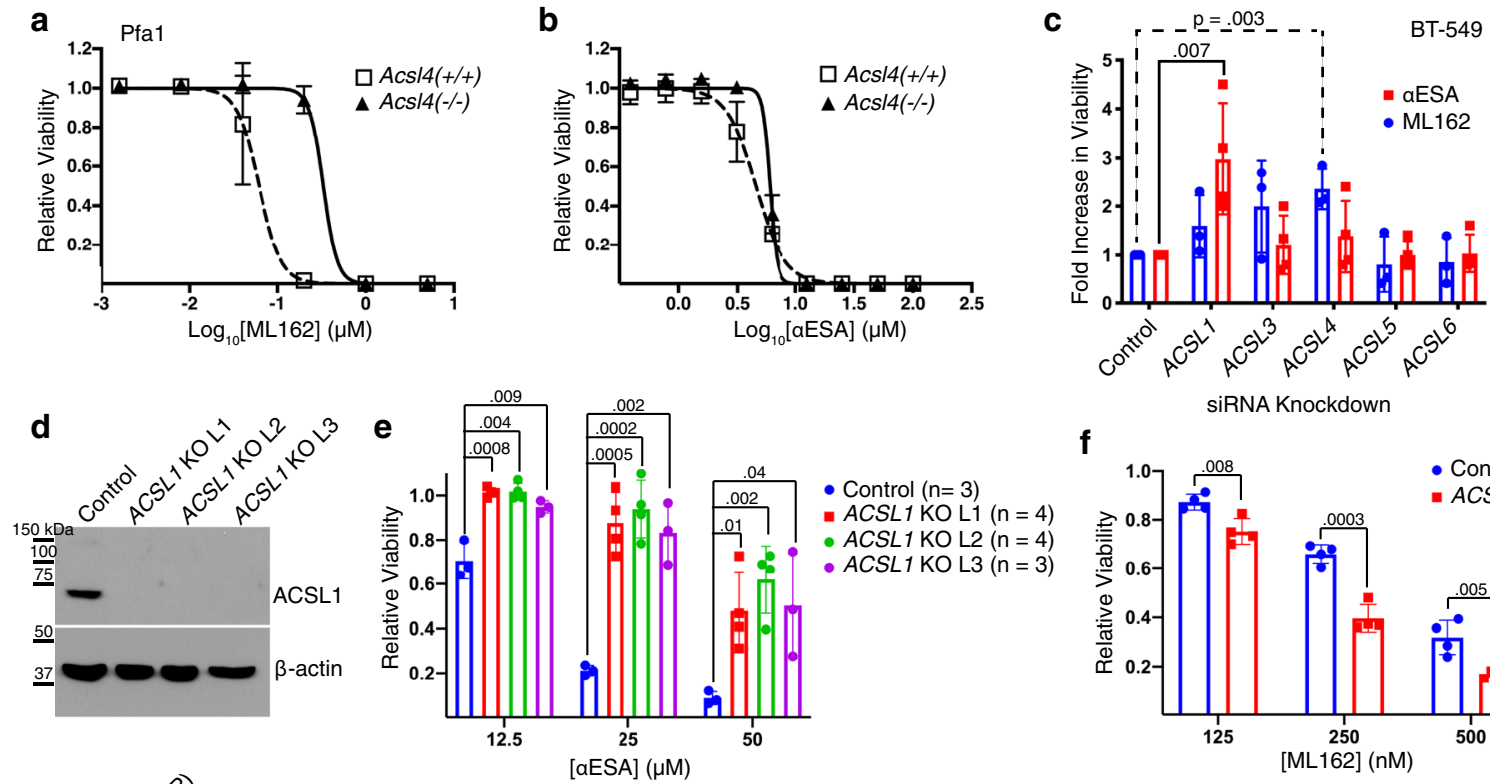

siRNA Knockdown

f
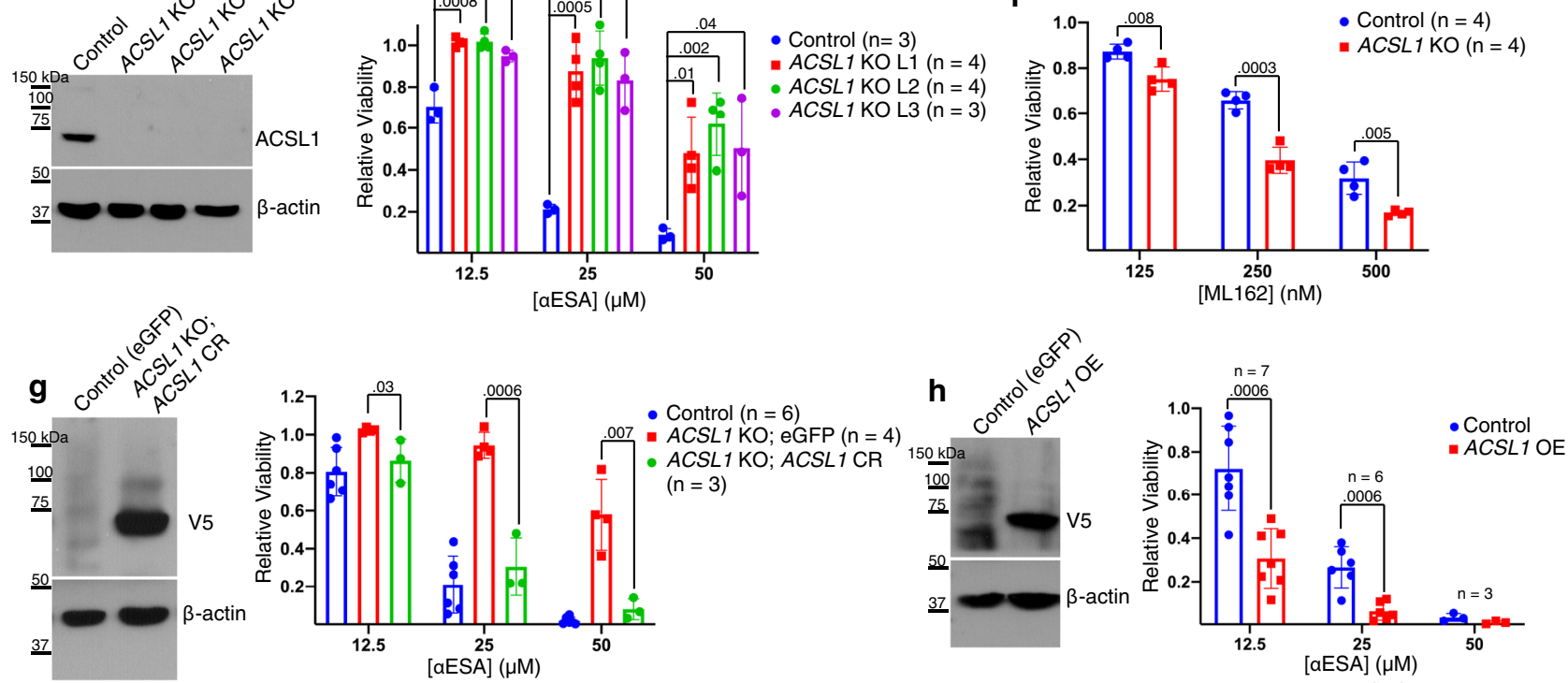

Fig. 5 ACSL1 mediates ferroptosis triggered by $\boldsymbol{\alpha E S A}$. Cell viability dose-response curves for control and ACSL4-deficient Pfa cells treated with a, ML162 or $\mathbf{b}, \alpha E S A$. Error bars indicate standard deviation from three independent experiments centered on the mean. $\mathbf{c}$ Bar charts showing fold change in the fraction of viable BT-549 relative to cells transfected with a non-targeting siRNA, $72 \mathrm{~h}$ after transfection with the designated ACSLI-targeted siRNA followed by 24-h treatment with either ML162 (blue, $n=3$ independent experiments) or $\alpha$ ESA (red, $n=4$ independent experiments). Error bars in this and subsequent panels show standard deviation centered on the mean. $p$ values $<0.05$ from Student's t-test (one-sided) are indicated. $\mathbf{d}$ Western showing ACSL1 protein levels in control BT-549 cells expressing a non-targeting guide RNA and three single-cell BT-549 clones in which ACSL1 was disrupted using CRISPR/Cas9 technology (ACSL1 KO L1-3). Similar results were observed using an independent ACSL1-targeting guide RNA (Supplementary Fig. 4g). e Relative cell viability of control and ACSL1 KO lines after $48 \mathrm{~h}$ of treatment with the specified dose of $\alpha$ ESA or $\mathbf{f}$, ML162 ( $n=4$ independent experiments). $\mathrm{p}$-values are shown above comparator bars in this and subsequent panels (two-sided Student's t-test). $\mathbf{g}$ V5 western blot (left) showing transgenic re-expression of V5-tagged, CRISPR-resistant ACSL1 (ACSL1 CR) and (right) relative viability of control (eGFP), ACSL1 KO (eGFP), or ACSL1 KO cells expressing ACSL1 CR after $48 \mathrm{~h}$ of treatment with the indicated dose of $\alpha$ ESA. $\mathbf{h}$ Western blot (left) of V5-tagged ACSL1 in BT-549 cells stably overexpressing the protein compared to a control line expressing eGFP, and (right) the fraction of viable cells remaining for each cell line after $48 \mathrm{~h}$ of treatment with the noted dose of $\alpha E S A$. Source data are provided as a Source Data file. 

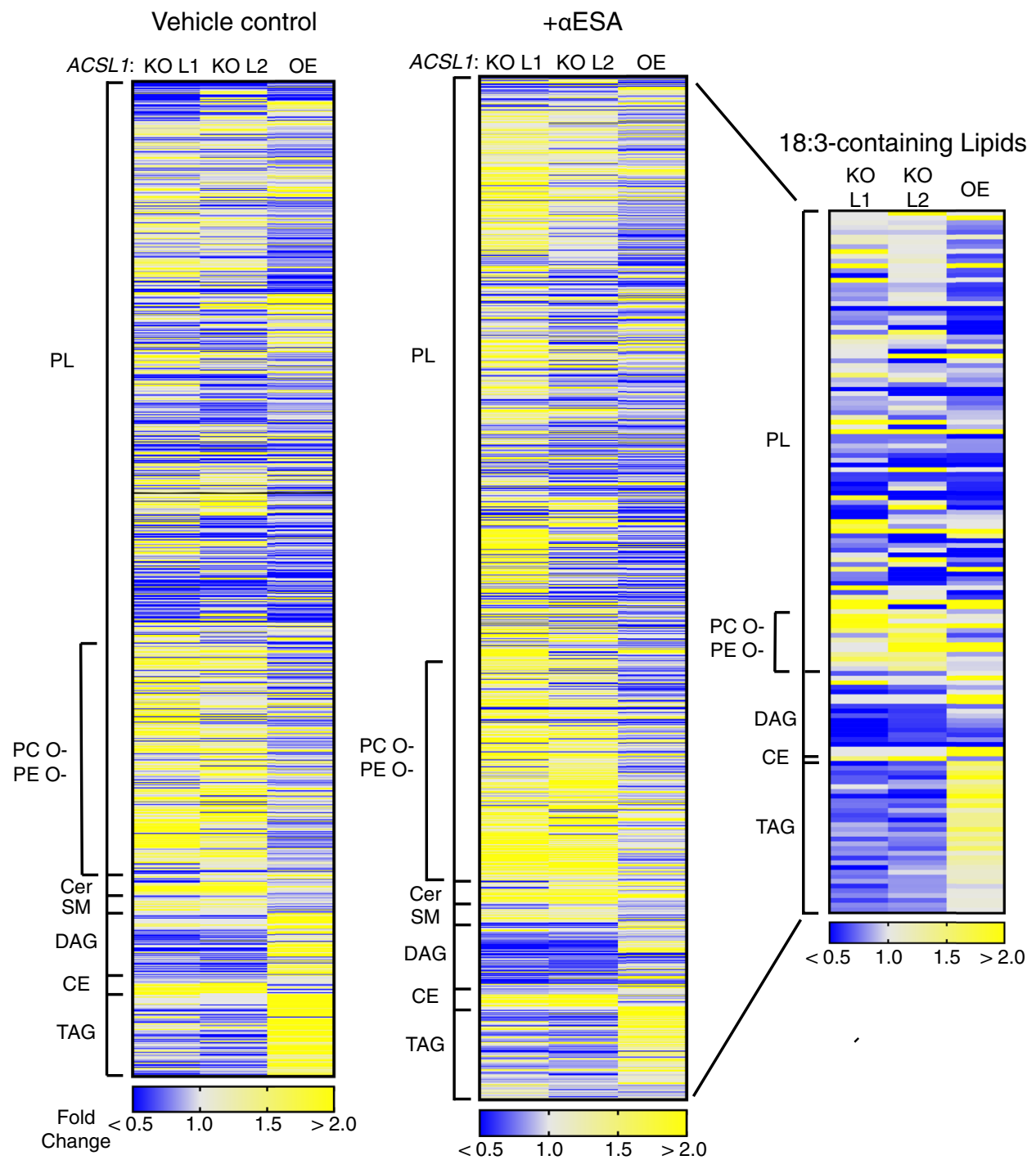

Fig. 6 ACSL1 drives incorporation of $\alpha$ ESA into neutral lipids. Heatmaps representing relative lipid abundance in ACSL1-deficient (ACSL1 KO L1, L2) or overexpressing ( $A C S L 1$ OE) BT-549 cells as fold-change in mole percent compared to control cells after $16 \mathrm{~h}$ treatment with $50 \mu \mathrm{M} \alpha \mathrm{ESA}$ (right and middle) or in untreated cells (left). The rightmost heatmap shows only the subset of lipids with 18:3 acyl chains. Yellow indicates an increase in relative abundance and blue shows a decrease in relative abundance. $\mathrm{PL}=$ phospholipids, $\mathrm{CE}=$ cholesterol esters, Cer $=$ ceramide, DAG $=$ diacylglycerol, $\mathrm{PC}$ O- $=$ ether-linked phosphatidylcholine, PE O- = ether-linked phosphatidylethanolamine, SM = sphingomyelin, TAG = triacylglycerol. Source data are provided as a Source Data file.

oxidation. First, we quantified 141 mono-, di-, and tri- oxygenated lipids using liquid chromatography-mass spectrometry across seven membrane lipid classes in BT-549 cells and found the abundance of oxidized membrane lipids was significantly increased in cells treated with $\alpha$ ESA $(p=0.02$, Student's t-test, one-tailed) (Fig. 7c). A wide variety of oxygenated lipids have been previously reported to be increased in cells undergoing ferroptosis, although a key role for phosphatidylethanolamine species bearing arachidonic or adrenic acids has been reported ${ }^{17}$. Within the seven lipid classes examined, we observed that $51(36 \%)$ oxygenated lipid species were significantly changed by aESA and a majority of those were increased $(41 / 51,80 \%)$ (Fig. 7c). These results are consistent with our observation of increased lipid peroxidation products in aESA-treated cells (Fig. 2d). The classes with both the highest number and frequency of increased oxidized species were cardiolipins (17/36 47\%) and phosphatidylethanolamines (18/40 45\%) and included two dioxygenated phosphatidylethanolamine species containing arachidonate residues (Fig. 7d, Supplementary Fig. 5).
Of the 41 oxygenated membrane lipids that were significantly increased following incubation with aESA, 10 (24\%) were responsive to ACSL1 silencing and all were decreased (Fig. 7e; Supplementary Fig. $5 c$, e, g, h). Some, though not all, aESAinduced, di-oxygenated phosphatidylethanolamine lipids were decreased on ACSL1-silencing (Fig. 7d). Given the abundant incorporation of aESA in TAGs, we also examined oxidation of this lipid class. Notably, in aESA-treated but not control cells we detected oxygenated 52:4 TAG (18:1/18:3/16:0) species including mono- and di-oxygenated species (Fig. $7 \mathrm{f}, \mathrm{g}$ ), consistent with a prior report ${ }^{28}$. However, few other oxidized TAGs were detected (Supplementary Data 4). The abundance of these species was decreased by ACSL1-silencing, as was the parental, unoxidized 52:4 TAG precursor (Supplementary Fig. 5i), demonstrating that these oxidized TAG species are generated in an aESA and ACSL1-dependent manner.

Triglycerides contribute to aESA toxicity. ACSL1 promotes aESA accumulation in DAGs and TAGs (Fig. 6) as well as aESA 


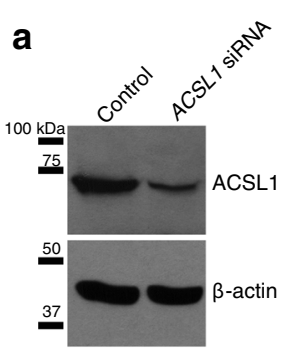

b

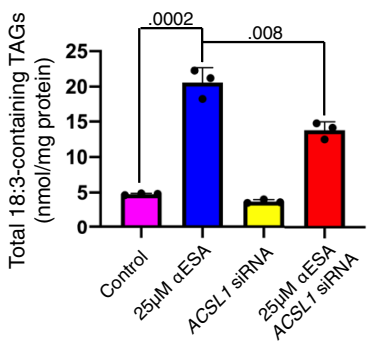

d
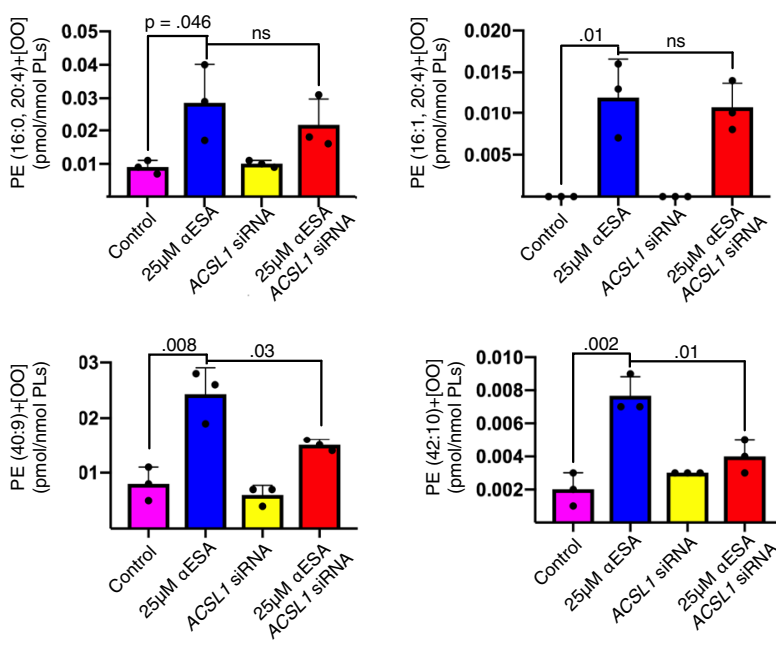

$\mathbf{f}$

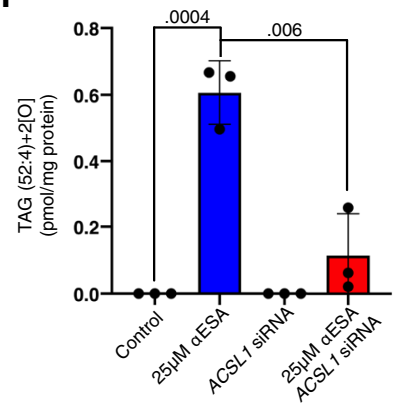

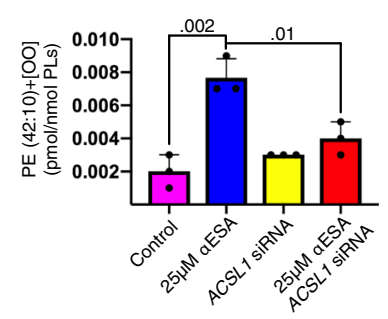

g

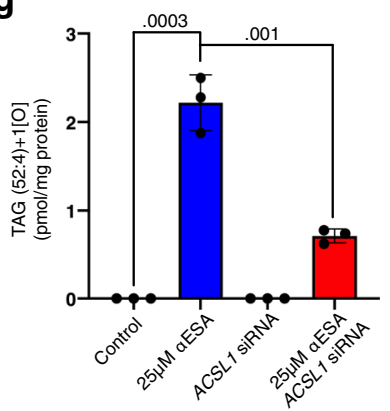

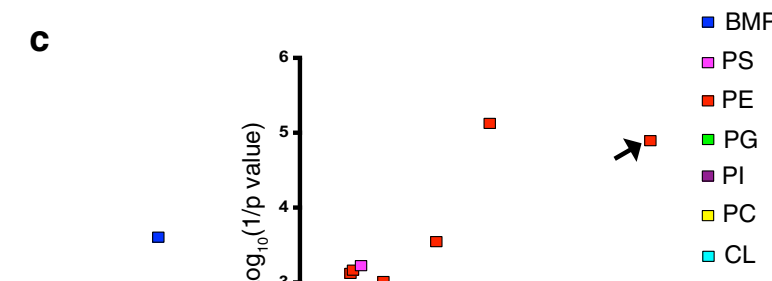

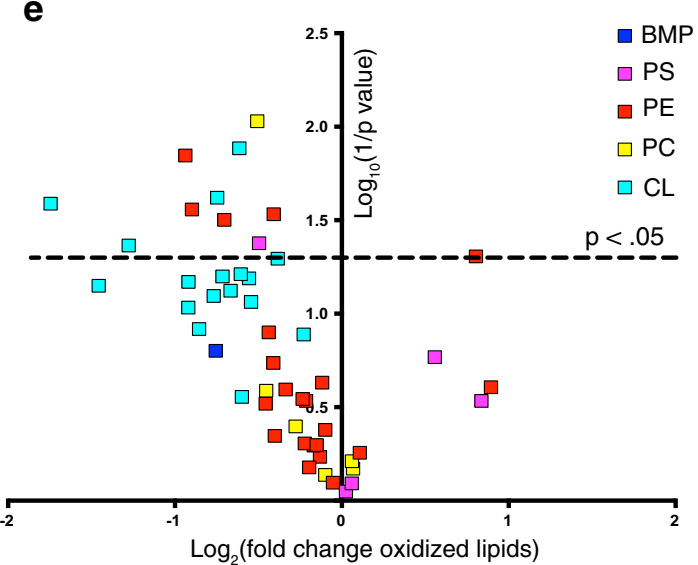

Fig. 7 Lipid peroxidation by $\boldsymbol{\alpha E S A}$ is promoted by ACSL1. a Western blot showing ACSL1 protein expression in control or ACSL1-silenced cells. The efficiency of the siRNA knockdown was independently confirmed using quantitative PCR ( amount of 18:3-containing TAGs in BT-549 cells under the specified condition ( $n=3$ biological replicates for this and subsequent bar charts). Cells were treated for $8 \mathrm{~h}$ with $25 \mu \mathrm{M} \alpha \mathrm{ESA}$ or vehicle. Treatments were added $72 \mathrm{~h}$ after transfection with a pool of either ACSL7-targeting or non-targeting siRNA. In this and subsequent panels, mean values \pm standard deviation are presented, and p-values are shown above comparator bars (two-sided Student's $t$-test). c Volcano plot showing $\log _{2}$ (fold change) and significance $\left(\log _{10}(1 / p)\right)$ for oxidized phospholipids in BT-549 cells incubated for $8 \mathrm{~h}$ with $25 \mu \mathrm{M} \alpha \mathrm{ESA}$ compared to cells incubated with vehicle (methanol). Each lipid species is colored according to phospholipid class. BMP = bis(monoacylglycero)phosphate, $\mathrm{PS}=$ phosphatidylserine, $\mathrm{PE}=$ phosphatidylethanolamine, $\mathrm{PG}=$ phosphatidylglycerol, $\mathrm{PI}=$ phosphatidylinositol, $\mathrm{PC}=$ phosphatidylcholine, $\mathrm{CL}=$ cardiolipin . The arrow indicates a lipid species for which a fold change could not be computed because it was only detected after $\alpha$ ESA treatment. $\mathbf{d}$ Bar charts showing the amounts of di-oxygenated PE species that were significantly increased by $\alpha$ ESA treatment compared to vehicle-treated controls. $\mathbf{e} V$ olcano plot depicts $\log _{2}$ (fold change) versus $\log _{10}(1 / p$-value) for oxidized phospholipids in BT-549 cells transfected with a pool of ACSL1 siRNA and incubated with $25 \mu M \alpha E S A$ for $8 \mathrm{~h}$ compared to $\alpha E S A$-treated cells transfected with non-targeting siRNA. The set is limited to the 51 oxidized phospholipid species that were significantly changed by $\alpha E S A$ treatment as shown in $\mathbf{c}$. Bar charts showing the amount of a (f), di-oxygenated and (g), mono-oxygenated TAG species that were significantly increased by $\alpha E S A$ treatment in an ACSL1-dependent manner. Source data are provided as a Source Data file.

toxicity (Fig. 5), suggesting a potential mechanistic link. We, therefore, examined the role of diacylglycerol acyl transferase (DGAT), which acylates DAGs to produce TAGs, in aESA toxicity. Transient silencing of DGAT1 but not DGAT2 restored viability of aESA-treated MDA-MB-231 cells (Fig. 8a, Supplementary Fig. 6a, b). Likewise, treatment of cells with the
DGAT1-specific inhibitor PF-04620110 but not the DGAT2specific inhibitor PF-06424439 suppressed aESA toxicity (Fig. 8b). Consistent with redundancy, the combination of both inhibitors produced an additive effect in MDA-MB-231 cells (Fig. 8b) and this combination also suppressed aESA-induced cell death in BT-549 cells (Fig. 8c). ML162 toxicity was not affected by 
a

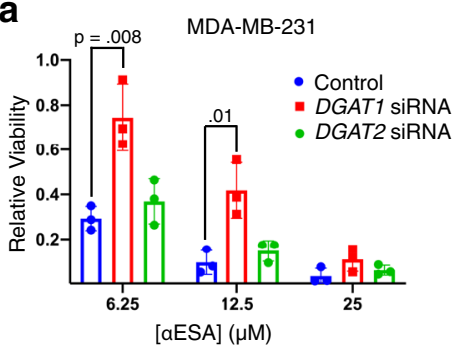

d

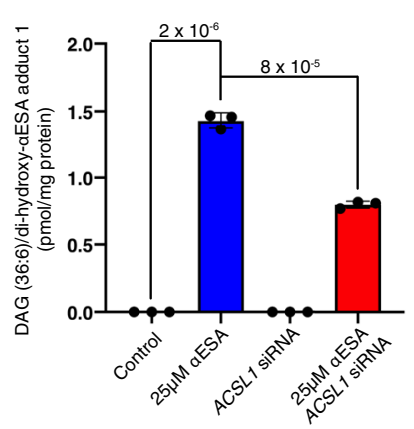

f

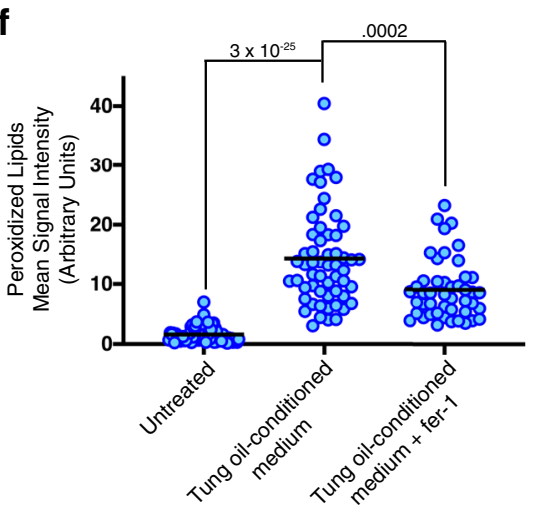

b

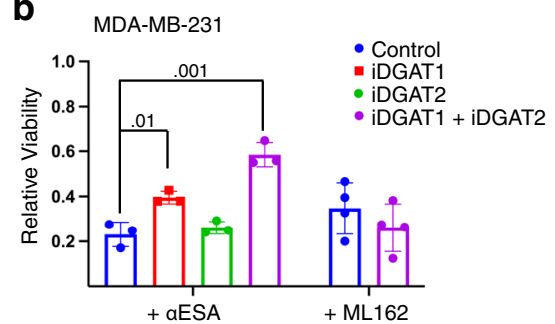

e

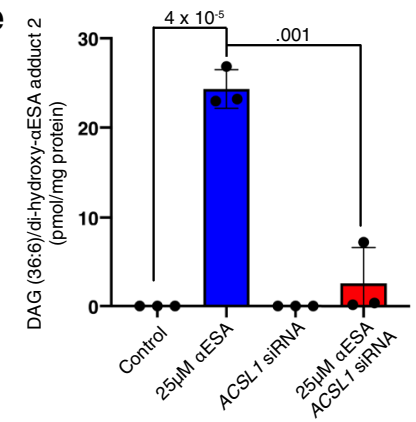

g

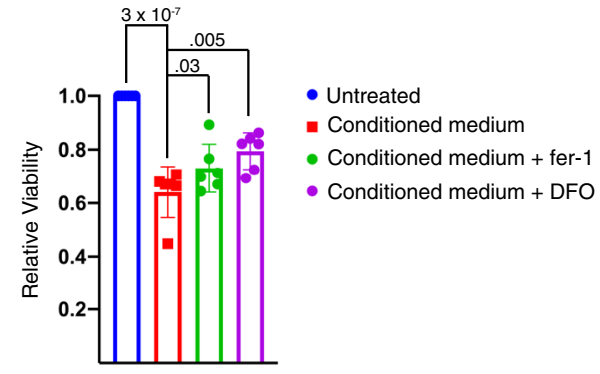

C

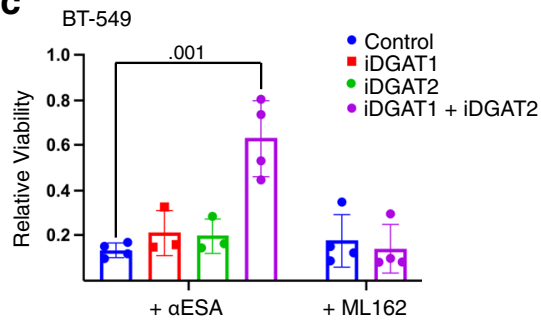

Fig. 8 Incorporation of $\boldsymbol{\alpha E S A}$ into triacylglycerols promotes ferroptosis. a Relative viability of MDA-MB-231 cells $72 \mathrm{~h}$ after transfection with either DGAT1-targeting, DGAT2-targeting, or non-targeting siRNA followed by 24-hour treatment with indicated dose of $\alpha$ ESA ( $n=3$ independent experiments). Error bars in this and subsequent panels show standard deviation centered on the mean, and p-values above comparator bars in this and subsequent panels are from two-sided Student's t-tests unless noted. Bar charts showing the fraction of viable (b), MDA-MB-231 or (c), BT-549 cells incubated for $48 \mathrm{~h}$ with either $6.25 \mu \mathrm{M} \alpha \mathrm{ESA}$ ( $n=3$ and 4 for MDA-MB-231 and BT-549, respectively) or $62.5 \mathrm{nM} \mathrm{ML162}(n=4)$ and either vehicle, a DGAT1 inhibitor ( $4 \mu \mathrm{M}$ PF04620110), a DGAT2 inhibitor (4 $\mu$ M PF-06424439), or both inhibitors. d, e Bar charts showing the amount of two DAG (18:3/18:3)/dihydroxy $\alpha$ ESA adducts that were detected following treatment with $\alpha E S A$ and were significantly decreased by ACSL1 depletion ( $n=3$ biological replicates). The $\mathrm{m} / z$ of adduct 1 and adduct 2 are 940.7278 and 942.7439 , respectively. $\mathbf{f}$ Quantitation of lipid peroxidation products in individual BT-549 cells after being cultured for $2 \mathrm{~h}$ with normal medium, tung oil-conditioned medium, or tung oil-conditioned medium containing $2 \mu \mathrm{M}$ fer-1. The line indicates the mean. From left to right, $n=71,57$, and 46. $\mathbf{g}$ Bar charts showing relative cell viability for BT-549 cells cultured in normal growth medium or tung oil-conditioned medium with or without $2 \mu \mathrm{M}$ fer- 1 or $50 \mu \mathrm{M}$ DFO ( $n=6$ independent experiments). The values above the comparator bars represent the $p$-values from one-sided Student's t-tests. Source data are provided as a Source Data file.

this inhibitor combination (Fig. 8b, c) indicating a role for TAGs specifically in aESA-induced ferroptosis. Knockdown of adipose triglyceride lipase (ATGL; Supplementary Fig. 6c), which liberates fatty acids from TAGs, suppressed the toxicity of ML162 but not aESA in two cell lines (Supplementary Fig. 6d-g). Atglistatin, a pharmacological inhibitor of ATGL, in contrast, suppressed both aESA and ML162 toxicity (Supplementary Fig. 6h-j), suggesting the possibility that atglistatin might act as a direct radical trapping agent as has been observed for other small molecule inhibitors ${ }^{42}$.

Fatty acids and TAGs with conjugated double bonds undergo complex and poorly understood oxidative reactions distinct from that of unsaturated chains with non-conjugated double bonds ${ }^{43-47}$. Products of conjugated acyl chain oxidation include aldehydes, peroxides, and high molecular weight covalent polymers ${ }^{45-49}$. Our mass spectrometry analysis of low molecular weight lipids from aESA-treated cells revealed the formation of two distinct adducts of di-oxygenated aESA free fatty acid with DAG containing two aESA-derived chains (Fig. 8d, e). These adducts were only detected in aESA-treated cells and were significantly reduced in ACSL1-knockdown cells, demonstrating an ACSL1-dependent formation of oxidative adducts of aESAderived acyl chains in neutral lipids. Higher-order polymers or adducts of aESA-containing TAGs were beyond the size range analyzed and thus our analysis likely underestimates the scope of TAG oxidation.

A remaining question is whether oxidative decomposition of aESA-derived acyl chains in neutral lipids could lead to the 
oxidation of membrane phospholipids. The seed oil of the tung tree is composed of TAGs in which $\sim 80 \%$ of the acyl chains are esterified aESA. We, therefore, considered tung oil as a model for the aESA-rich TAGs that accumulate in aESA-treated cells (Fig. 2g). We generated an emulsion of tung oil in cell culture medium and, following sedimentation and removal of the oil layer, incubated the conditioned medium with BT-549 cells. Two hours in conditioned medium led to an increase in cellular lipid peroxidation products, which were suppressed by co-treatment with fer-1 (Fig. 8f). Incubating cells with conditioned medium for $24 \mathrm{~h}$ resulted in significantly decreased cell viability, which was partially rescued by fer-1 or iron chelation by deferoxamine (Fig. 8g). These findings demonstrate that aESA-rich triglycerides can produce a diffusible mediator of ferroptosis that could potentially propagate lipid peroxidation from neutral lipids to membrane lipids.

Conjugated PUFAs exhibit anti-cancer activity and promote expression of ferroptotic markers in vivo. Finally, we assessed the anti-cancer activity of tung oil in an aggressive TNBC orthotopic xenograft model. Mice with established tumors were treated by oral gavage five times per week with $100 \mu \mathrm{l}$ tung oil. Control mice received high-oleic (18:1) safflower oil. Body weights of tung oil-treated mice did not differ significantly from controls (Supplementary Fig. 7a). However, tung oil significantly suppressed tumor growth (Fig. 9a) and endpoint tumor weight (Fig. 9b) and also substantially reduced lung metastatic invasion compared to controls (Fig. 9c, d). The addition of BSO further enhanced tumor growth suppression by aESA (Supplementary Fig. $7 \mathrm{~b}$ ), consistent with BSO enhancing $\alpha \mathrm{ESA}$ toxicity in vitro (Fig. 2b).

To obtain evidence that the reduction in tumor growth is associated with tumor exposure to $\alpha E S A$, we conducted a lipidomic analysis of aESA-treated and control tumors. The two most differentially expressed lipids were DAG (34:3) and TAG (52:7), which were increased in tung oil-treated tumors by $30 \%$ and $60 \%$, respectively (Fig. 9e). The masses of these lipids are consistent with at least one linolenate moiety, although their precise acyl chain composition was not determined. Notably, the expression of these two lipids was also increased in aESA-treated cultured cells (Fig. 9e). Thus, increased DAG (34:3) and TAG (52:7) could reflect tumor exposure to circulating aESA released from tung oil and taken up by tumor cells.

Next, we assessed whether tumors in aESA-treated mice expressed markers of ferroptosis. We conducted RNA sequencing of three tumors each from treated and control mice and compared the differentially expressed genes with a separate in vitro experiment using cultured MDA-MB-231 cells treated with either $\alpha$ ESA, the GPX4 inhibitor ML162, or vehicle controls. Using a false discovery rate of $5 \%$ and a filter of $>2$-fold change in RNA expression, 124 genes were altered in tung oil-treated tumors compared to safflower oil-treated controls (Fig. 9f). Significantly, 89 genes $(89 / 122,73 \%)$ altered in tung oil-treated tumors were also altered in aESA-treated cells in culture. Fisher's exact test showed significant overlap between these gene sets $(p=$ $3.2 \times 10^{-246}$ ) and all 89 overlapping genes were altered in the same direction. This is consistent with our hypothesis that the inhibition of tumor growth in vivo is related to the cell death caused by $\alpha E S A$ in vitro. Importantly, there was also significant overlap in genes altered by tung oil in vivo, $\alpha \mathrm{ESA}$ in vitro, and by in vitro treatment with ML162 (Fig. 7f). Fisher's exact test between any two gene sets demonstrated $p$-values of $<7.2 \times 10^{-61}$. Furthermore, all of the shared genes were altered in the same direction. These results demonstrate concordant gene signatures between tung oil treatment and aESA treatment, consistent with the hypothesis that tumors in tung oil-treated mice are exposed to aESA. Furthermore, the overlap in the signatures of aESA- and ML162-treated cells provide strong evidence that both treatments trigger a similar ferroptotic response.

All three treatments altered a common set of 23 genes (Fig. 9f) including genes previously identified as increased during ferroptosis (RHOB, SLC2A3, DDIT4, and HMOX1 $)^{50,51}$. In addition, the previously reported ferroptosis marker $C H A C 1^{50}$ was commonly upregulated in both tung-oil treated tumors and aESA-treated cells. 66 genes were altered by both $\alpha$ ESA and tung oil but not ML162, suggesting a potential source of biomarkers for aESA-induced ferroptosis.

\section{Discussion}

While anti-cancer activity has been previously attributed to various conjugated linolenic acids including $\operatorname{aESA}^{52,53}$, here we reveal the mode of action as ferroptosis and highlight mechanistic differences compared to existing ferroptosis inducers. Our findings suggest that while GPX4 inhibitors promote ferroptosis by inhibiting the glutathione-dependent reduction of hydroperoxides, aESA promotes ferroptosis by enhancing hydroperoxide production. GPX4 activity reduces aESA-induced hydroperoxide accumulation but ultimately GPX4 is overwhelmed.

We found that ACSL1 mediates aESA-induced ferroptosis as well as aESA incorporation into specific lipid species including DAGs and TAGs (Figs. 5, 6 and 7b). Further supporting a role for TAGs in ferroptosis, depletion or pharmacological inhibition of DGAT activity suppressed aESA toxicity (Fig. $8 \mathrm{a}-\mathrm{c}$ ). We found that ACSL1, which is constitutively localized to lipid droplets ${ }^{54}$ concentrates aESA in neutral lipids compared to other lipid pools. By three hours of treatment with aESA, TAG levels increase 2-fold (Fig. $2 \mathrm{~h}$ ) with $>70 \%$ containing at least one 18:3 acyl chain while no membrane phospholipid class has more than $20 \%$ (Fig. $2 \mathrm{~g}$ ). We hypothesize that neutral lipids bearing aESAderived acyl chains undergo spontaneous or enzyme-mediated oxidation after achieving a critical concentration in lipid droplets. This concentration dependence could reflect the intermolecular nature of the rate-limiting propagation step of lipid autoxidation. The peroxy radicals that mediate the chain-reaction propagation phase of autoxidation either abstract a hydrogen atom from adjacent hydroperoxides or form intermolecular covalent bonds by adding to carbon-carbon double bonds. The rate of abstraction of a hydrogen atom adjacent to the triene system of conjugated linolenic acid is 2.5 -fold faster than the corresponding rate for abstraction of the bis-allylic hydrogen in non-conjugated linolenic acid ${ }^{43}$. Furthermore, peroxyl radicals also add to the triene system of conjugated linolenic acid at an even faster rate while peroxyl radicals do not appreciably add to double bonds in nonconjugated linolenic acid. This results in an overall rate of free radical oxidation propagation that is over 8-fold faster for conjugated linolenic acid ${ }^{43}$, providing a chemical basis for the distinct ferroptosis-inducing activity of this lipid class above a threshold concentration.

While we only detected two oxidized TAG species associated with aESA treatment (Fig. 7f, g), we speculate that neutral lipids containing esterified $\alpha \mathrm{ESA}$ undergo oxidative polymerization to form higher molecular weight polymers, as has been previously shown ex vivo ${ }^{4-48}$. Because we focused our analysis on lipids $<1,200$ molecular weight, very large species, and any oxygenated derivatives were not examined by our lipidomic analysis. However, our demonstration of adducts of free aESA with DAGs containing esterified aESA (Fig. 8d, e), which fall under this size cutoff, supports the possibility that larger covalent aggregates may also form. 
a

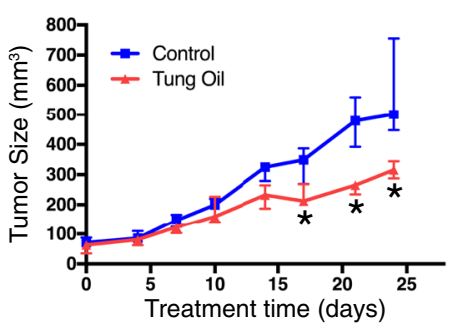

c

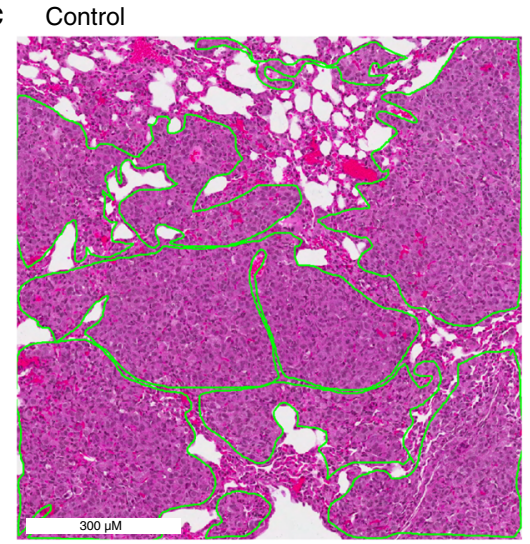

b

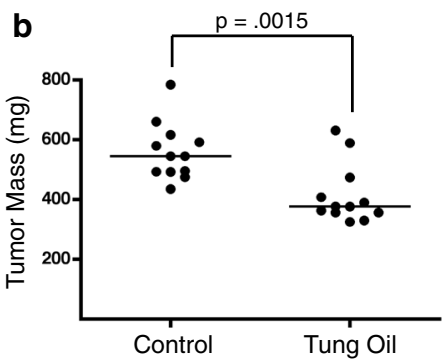

Tung Oil

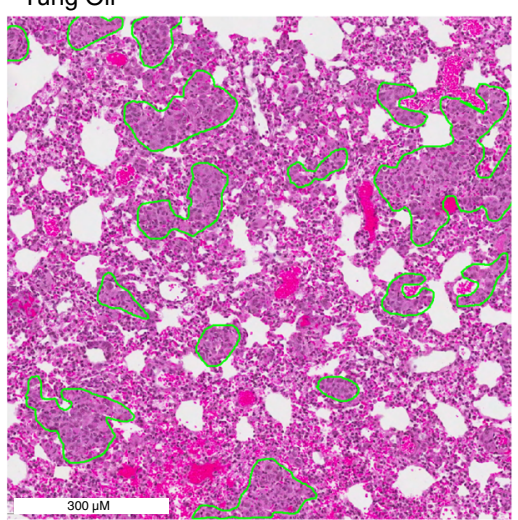

d

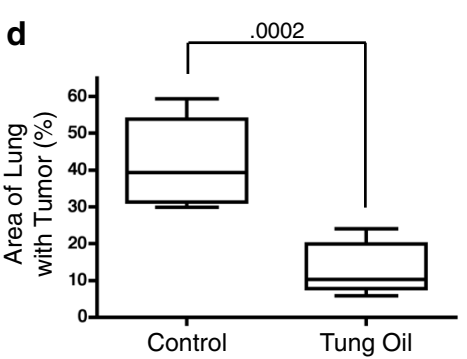

e

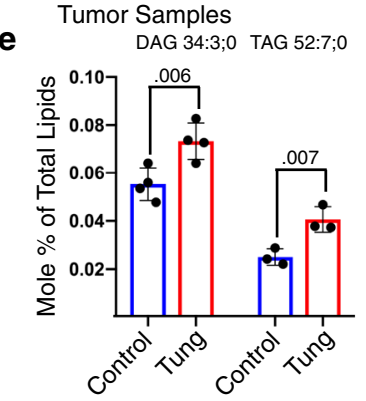

Cell Culture Samples

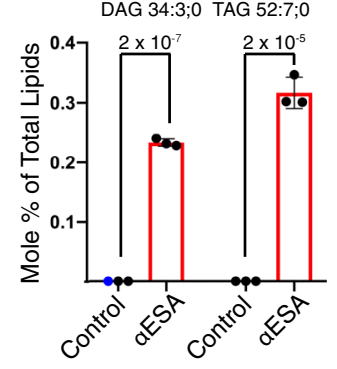

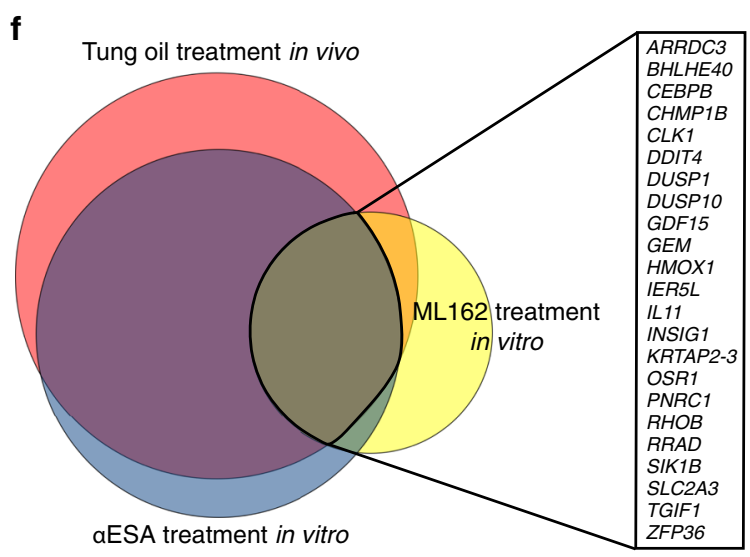

A role for lipid droplets in potentiating lipid peroxidation is contrary to a proposed protective role for this organelle against oxidative stress ${ }^{55,56}$. This may be explained, however, by the rapid rate of free radical oxidation propagation by conjugated linolenic acids. Dietary conjugated linoleic acids have been linked to increased lipid peroxidation in humans $s^{57,58}$ and animal models ${ }^{59}$, and $\alpha \mathrm{ESA}$ exhibits even more rapid spontaneous oxidation than these species ${ }^{33,60}$. While lipid droplets may protect polyunsaturated lipids from oxidation by sequestering them from ROS species generated elsewhere, the accumulation of esterified conjugated linolenic acids, which are not naturally synthesized by mammals, in lipid droplets may promote their oxidation and polymerization there. Propagation of lipid peroxidation from lipid droplets to membrane phospholipids may be mediated by diffusion of soluble mediators (Fig. 8f, g). The formation of large covalent lipid polymers in lipid droplets may also trigger an autophagic response ${ }^{61}$ which could drive ferroptosis ${ }^{62}$.

A model in which accumulation of conjugated linolenic acyl chains in lipid droplets initiates ferroptosis may also explain the differences in ACSL isoform roles between ferroptosis triggered by aESA and by GPX4 inhibitors. GPX4 inhibitors suppress the antioxidant response, allowing natural lipid ROS species to 
Fig. 9 Tung oil suppresses TNBC xenograft growth and metastasis with markers of ferroptosis. a Median tumor volumes over time for orthotopic MDAMB-231 xenografts in NSG mice treated orally with either safflower oil (control) or tung oil ( $n=6$ mice per group with bilateral tumors). * denote $p$-values from two-sided Student's $t$-test, which are $0.003,0.00001$, and 0.0003 from left to right. Error bars show interquartile range. b Final tumor masses of individual tumors following 24 days of treatment. Lines represent median values. The $p$-value above the comparator bar is from a two-sided Student's $t$-test. c Representative hematoxylin and eosin-stained lung sections. Regions containing metastatic TNBC cells are outlined in green. $\mathbf{d}$ Quantification of the percentage of lung area infiltrated by metastatic cells. The line represents the median value, the box shows the interquartile range (25th to 75 th percentile), and the whiskers show minimum and maximum values. This analysis was performed once. The $p$-value above the comparator bar is from a two-sided Student's $t$-test. e Mole percent of total lipids for DAG 34:3 and TAG 52:7 in tumors treated with tung oil (left, $n=4$ and 3 biological replicates for DAG 34:3 and TAG 52:7, respectively) or in MDA-MB-231 cells grown in culture and incubated with $50 \mu \mathrm{M} \alpha \mathrm{ESA}$ for $3 \mathrm{~h}$ (right, $n=3$ biological replicates). Error bars represent standard deviation centered on the mean. The $p$-values were calculated using a one-sided Student's $t$-test, not adjusted for multiple hypothesis testing. f Proportional Venn diagram showing the overlap in genes with transcript levels that were significantly altered by $>2$-fold (5\% false discovery rate) after a $5 \mathrm{~h}$ incubation with $100 \mu \mathrm{M} \alpha \mathrm{ESA}$ or $125 \mathrm{nM} \mathrm{ML-162}$ or in orthotopic MDA-MB-231 xenograft tumors treated as described above for a. Genes commonly altered in all three data sets are listed. Source data are provided as a Source Data file.

accumulate. In many contexts these are likely enzymatically generated peroxidation products of arachidonyl and adrenyl acyl chains, whose incorporation into membrane phospholipids is mediated by ACSL4 ${ }^{38}$. By contrast, the ACSL1-dependence of aESA toxicity appears to be mediated at least in part by ACSL1driven incorporation of aESA into TAGs. Overall, these findings suggest that conjugated linolenic acids trigger ferroptosis by a mechanism that is mediated by ACSL1 and support emerging evidence implicating lipid composition as a critical determinant of ferroptotic sensitivity ${ }^{63}$.

The discrepant ability of GPX4 and FSP1 to suppress aESAtriggered ferroptosis compared to ferroptosis induced by ML162 in BT549 cells (Fig. 4g-j) could also be explained by distinct origins of lipid peroxidation. We speculate that GPX4 and FSP1 may have evolved and be localized specifically to target natural sources of lipid ROS. Indeed, FSP1 is N-myristoylated and is localized to intracellular membranes and the plasma membrane in a cell type-specific manner ${ }^{9,10}$. Plasma membrane-restricted FSP1 restored RSL3-resistance to FSP1-deficient cells while FSP1 targeted to other intracellular locations did not ${ }^{10}$, suggesting that the plasma membrane is an important site of lipid radical accumulation on GPX4 inhibition. aESA, by contrast, is metabolized like other nutrient fatty acids and stored in lipid droplets where, because of its oxidizable conjugated triene system, it may act as a source of lipid ROS that is poorly targeted by endogenous protective mechanisms. This concept has clear applicability to targeting cancer cells that robustly scavenge extracellular fatty acids $^{22}$.

Studies have shown that cancer cells, including renal cell carcinoma, melanoma, and glioblastoma are vulnerable to ferroptosis ${ }^{38,51,64-68}$. Furthermore, therapy-resistant mesenchymal cancer cells exhibit a greater reliance on GPX4 activity for survival ${ }^{64,69}$. These findings have highlighted the therapeutic potential of ferroptosis, but it remains unclear whether induction of ferroptosis can be used to kill cancer cells in vivo ${ }^{1}$. A major challenge to testing this hypothesis has been the absence of ferroptosis-inducing agents, such as GPX4 inhibitors, suitable for use in vivo ${ }^{70}$. Here, we found that treatment of mice with tung oil, a rich source of aESA, suppressed tumor growth and metastasis, and resulted in gene expression changes in tumors consistent with ferroptosis. The data suggest that GPX4 inhibitors, should these become clinically viable, may enhance the anti-tumor activity of aESA. Thus, these findings expand our understanding of the pathways that regulate ferroptosis and provide an approach for exploiting ferroptosis therapeutically.

\section{Methods}

Cell lines. MDA-MB-468 cells were obtained by the Cell Culture Facility at Fox Chase Cancer Center from ATCC (HTB-132) as part of the NCI-60 panel. MDAMB-231 (HTB-26), BT-20 (HTB-19), BT-549 (HTB-122), HCC38 (CRL-2314),
HCC1806 (CRL-2335), HCC1187 (CRL-2322), HCC1143 (CRL-2321), HCC70 (CRL-2315), Hs-578T (HTB-126), cell lines and the MCF-10A cell line (CRL10317) were obtained directly from the American Type Culture Collection (ATCC, Manassas, VA 20110, USA). Note: BT-20 has been identified as a frequently misidentified cell line by the ICLAC Register of Misidentified Cell Lines but ATCC maintains the authentic stock (HTB-19). The suppliers routinely authenticate the cell lines by short tandem repeat profiling though only MDA-MB-231 cell lines were authenticated by our laboratory (April 2018). MCF-12A cells were a kind gift of Dr. Jose Russo. All cell lines were amplified and frozen within 2 months of receipt. TNBC cell lines were cultured in RPMI-1640, 10\% heat inactivated fetal bovine serum (FBS), $2 \mathrm{mM}$ supplemental glutamine, and $100 \mu \mathrm{g} / \mathrm{mL}$ penicillin/ streptomycin $(\mathrm{P} / \mathrm{S})$ with the exception of BT-20, which was cultured in MEM with the same supplements. MCF-10A and MCF-12A cells were cultured in high calcium medium with $5 \%$ horse serum as described ${ }^{71}$. Lenti-X 293 T cells were cultured in DMEM plus 10\% FBS, $2 \mathrm{mM}$ glutamine, and P/S. The FBS content of the medium was increased to $30 \%$ during lentiviral production. Mouse embryonic fibroblast (Pfa1) Acsl4-deficient and control cells were cultured as described previously ${ }^{38}$. Cells were cultured in a humidified incubator at $37^{\circ} \mathrm{C}$ with $5 \% \mathrm{CO}_{2}$. Cell lines are periodically tested for Mycoplasma contamination using DAPI (4',6-diamidino-2-phenylindole) to stain DNA.

PRISM 100 cell line viability profiling. Cell viability across a panel of 100 human cancer cell lines at 6 doses for each of 14 fatty acids was determined using the multiplexed PRISM screening platform ${ }^{34}$. Quantitative cell line bar code data from cells treated for $48 \mathrm{~h}$ with the indicated fatty acid (in triplicate at 5-6 doses ranging from 0 to $35 \mu \mathrm{M}$ ) were scaled from 0 (no viable cells) to 1 (full viability) based on median values from DMSO-treated control wells and wells treated with saturating doses of the death-inducing control compound bortezomib. Rescaled dosedependent cell viability data were fitted to a three-parameter log-logistic model and Activity Area (reflecting cell line-specific toxicity) was calculated using Thunor ${ }^{35}$ for each combination of fatty acid and cell line. A histogram reflecting the range of Activity Areas calculated across the cell line panel for each fatty acid is presented as a violin plot in Fig. 3b. Pearson correlation coefficients were calculated for the Activity Areas across the cell line panel for each pair of fatty acids. The heatmap shown in Fig. $3 c$ was generated using GraphPad Prism (Version 8).

Statistical Analysis. Metabolite profiling data shown in Fig. $1 \mathrm{~h}$ and i were $\log _{10^{-}}$ transformed before further analysis to achieve an approximate normal distribution. Missing values were not imputed for univariate analysis. Mixed model analysis of variance (ANOVA) using $\mathrm{R}$ with package nlme was applied to identify differentially expressed intracellular metabolites in non-ferroptotic (HCC38, HCC1806, HCC1143, HCC70) compared to ferroptotic (MDA-MB-468, MDA-MB-231, BT549, BT-20, Hs-578T) TNBC cell lines. Our previously reported data set of 155 metabolites including 5-6 replicates per cell line ${ }^{23}$ was taken considering "cell line" as a random factor and "ferroptosis response" as categoric factor. ANOVA models were read out concerning t-statistics results comprising estimates, $t$-values, and p-values. Significance level was set to an $\alpha$-error of 5\%. The multiple test problem was addressed by calculating the false discovery rate (FDR) using the Benjamini \& Hochberg method. For the lipidomic data presented in Fig. 6 and Supplementary Data 2 and 3, combined p-values were calculated using Fisher's combined probability test, and the significance threshold was corrected using the Benjamini \& Hochberg method and 5\% FRD. In general, other statistical comparisons were performed using Student's t-test unless noted and the threshold for significance was $p<0.05$. Data are reported as mean and standard deviation of at least $2-3$ independent experiments unless otherwise stated. All data points reflect measurements of distinct samples.

Lipidomic analysis. For Fig. 2g, h, triplicate samples of MDA-MB-231 cells at $\sim 60 \%$ confluence were incubated with vehicle (methanol), $2 \mu \mathrm{M}$ fer- $1,50 \mu \mathrm{M} \alpha \mathrm{ESA}$ $50 \mu \mathrm{M} \alpha \mathrm{ESA}$ and $2 \mu \mathrm{M}$ fer-1, or $250 \mathrm{nM}$ ML162 for $3 \mathrm{~h}$ and then were typsinized, 
washed twice with phosphate buffered saline (PBS) without magnesium or calcium, and resuspended at a concentration of 1500 cells $/ \mu \mathrm{L}$, and frozen in liquid nitrogen. For Fig. 6, three replicates of two independent ACSL1-deficient BT-549 cell lines, a stably over-expressing ACSL1 line, or a control line expressing a non-targeting guide were incubated with either vehicle (methanol) or $50 \mu \mathrm{M}$ aESA (Cayman Chemical) for $16 \mathrm{~h}$ and then prepared as above. Cells of a different passage number were used for each replicate. Plates were approximately $70-80 \%$ confluent at the time of harvest. For tumor samples (Fig. 9e), four biological replicate tumor samples per condition were homogenized in PBS without magnesium or calcium using a dounce homogenizer to yield a sample containing $5 \mathrm{mg}$ of tumor (wet weight $) / \mathrm{mL}$ and were then frozen in liquid nitrogen.

Mass spectrometry (MS)-based lipid analysis in Figs. 2g, h, 6, and 9e were performed by Lipotype GmbH (Dresden, Germany) as described ${ }^{72}$. Lipids were extracted using a two-step chloroform/methanol procedure ${ }^{73}$. Samples were spiked with internal lipid standard mixture containing: cardiolipin 16:1/15:0/15:0/15:0 (CL), ceramide 18:1;2/17:0 (Cer), diacylglycerol 17:0/17:0 (DAG), hexosylceramide 18:1;2/12:0 (HexCer), lyso-phosphatidate 17:0 (LPA), lyso-phosphatidylcholine 12:0 (LPC), lyso-phosphatidylethanolamine 17:1 (LPE), lyso-phosphatidylglycerol 17:1 (LPG), lyso-phosphatidylinositol 17:1 (LPI), lyso-phosphatidylserine 17:1 (LPS), phosphatidate 17:0/17:0 (PA), phosphatidylcholine 17:0/17:0 (PC), phosphatidylethanolamine 17:0/17:0 (PE), phosphatidylglycerol 17:0/17:0 (PG), phosphatidylinositol 16:0/16:0 (PI), phosphatidylserine 17:0/17:0 (PS), cholesterol ester 20:0 (CE), sphingomyelin 18:1;2/12:0;0 (SM), triacylglycerol 17:0/17:0/17:0 (TAG). After extraction, the organic phase was transferred to an infusion plate and dried in a speed vacuum concentrator. The dried extract was re-suspended in $7.5 \mathrm{mM}$ ammonium acetate in chloroform/methanol/propanol (1:2:4, V:V:V) and the second step dry extract was re-suspended in a $33 \%$ ethanol solution of methylamine in chloroform/methanol $(0.003: 5: 1 ; \mathrm{V}: \mathrm{V}: \mathrm{V})$. All liquid handling steps were performed using the Hamilton Robotics STARlet robotic platform. Samples were analyzed by direct infusion on a QExactive mass spectrometer (ThermoFisher Scientific) equipped with a TriVersa NanoMate ion source (Advion Biosciences). Samples were analyzed in both positive and negative ion modes with a resolution of $\mathrm{Rm} / \mathrm{z}=200=280000$ for MS and $\mathrm{Rm} / \mathrm{z}=200=17500$ for MS-MS experiments, in a single acquisition. MS-MS was triggered by an inclusion list encompassing corresponding MS mass ranges scanned in $1 \mathrm{Da}$ increments ${ }^{74}$. Both MS and MSMS data were combined to monitor CE, DAG, and TAG ions as ammonium adducts; PC, PC O-, as acetate adducts; and CL, PA, PE, PE O-, PG, PI and PS as deprotonated anions. MS only was used to monitor LPA, LPE, LPE O-, LPI and LPS as deprotonated anions; Cer, HexCer, SM, LPC, and LPC O- as acetate adducts. Data were analyzed using lipid identification software based on LipidXplorer ${ }^{75,76}$. Data post-processing and normalization were performed using an in-house developed data management system (Lipotype). Only lipid identifications with a signal-to-noise ratio $>5$, and a signal intensity 5 -fold higher than in corresponding blank samples were considered for further data analysis. To approximate the mole percent of triacylglycerol species containing aESA-derived acyl chains, triacylglycerols with at least three desaturations that were significantly increased above the detection limit, or increased greater than 2-fold compared to control cells after aESA treatment, were considered to contain esterified aESA. The heatmap shown in Fig. 6 was generated using GraphPad Prism (Version 8). Lipids below the detection limit were considered to have a mole percent of zero.

\section{Quantification of oxidized cellular phospholipids and triacylglycerols (TAGs)}

BT-549 cells were transfected with ACSL1 or non-targeting siRNA $72 \mathrm{~h}$ prior to incubation with $25 \mu \mathrm{M}$ aESA or vehicle (methanol) for $8 \mathrm{~h}$. After $8 \mathrm{~h}$, cells $(\sim 4-6 \times$ $10^{6}$ per replicate) were trypsinized, collected, washed once with phosphate-buffered saline, and frozen in liquid nitrogen. Triplicate samples were collected for each condition. MS analysis of PLs was performed on an OrbitrapTM FusionTM LumosTM mass spectrometer (ThermoFisher Scientific). PLs were separated on a normal phase column (Luna $3 \mu \mathrm{m}$ Silica (2) $100 \AA ., 150 \times 2.0 \mathrm{~mm}$, (Phenomenex)) at a flow rate of $0.2 \mathrm{~mL} / \mathrm{min}$ on a Dionex Ultimate $3000 \mathrm{HPLC}$ system. The column was maintained at $35^{\circ} \mathrm{C}$. The analysis was performed using gradient solvents (A and B) containing $10 \mathrm{mM}$ ammonium formate. Solvent A contained propanol: hexane:water (285:215:5, v/v/v) and solvent B contained propanol:hexane:water (285:215:40, v/v/v). All solvents were LC/MS grade. The column was eluted for $0-23$ min with a linear gradient from 10 to $32 \% \mathrm{~B} ; 23-32$ min using a linear gradient of $32-65 \% \mathrm{~B} ; 32-35$ min with a linear gradient of $65-100 \% \mathrm{~B} ; 35-62 \mathrm{~min}$ held at $100 \%$ B; 62-64 min with a linear gradient from 100 to $10 \%$ B followed by an equilibration from 64 to $80 \mathrm{~min}$ at $10 \% \mathrm{~B}$. The instrument was operated with ESI probe in negative polarity mode. Analysis of LC/MS data was performed using software package Compound Discoverer (ThermoFisher Scientific) with an inhouse generated analysis workflow and oxidized phospholipid database.

LC/ESI-MS analysis of TAGS was performed on a Dionex HPLC system (utilizing the Chromeleon software), consisting of a Dionex UltiMate 3000 mobile phase pump, equipped with an UltiMate 3000 degassing unit and UltiMate 3000 autosampler (sampler chamber temperature was set at $4^{\circ} \mathrm{C}$ ). The Dionex HPLC system was coupled to a hybrid quadrupole-orbitrap mass spectrometer, Q-Exactive (ThermoFisher) with the Xcalibur operating system. The instrument was operated in the positive ion mode (at a voltage differential of $-5.0 \mathrm{kV}$, source temperature was maintained at $\left.150^{\circ} \mathrm{C}\right)$. TAG cations were formed through molecular ammonium adduction $\left(+\mathrm{NH}_{4}\right)$. Analysis was performed at a resolution of 140,000 for the full MS scan and 17,500 for the MS ${ }^{2}$ scan in a data-dependent mode. The scan range for MS analysis was $300-1200 \mathrm{~m} / \mathrm{z}$ with a maximum injection time of $128 \mathrm{~ms}$ using one microscan. A maximum injection time of $500 \mathrm{~ms}$ was used for $\mathrm{MS}^{2}$ (high energy collisional dissociation (HCD)) analysis with collision energy set to 24 . An isolation window of $1.0 \mathrm{Da}$ was set for the MS and $\mathrm{MS}^{2}$ scans. Capillary spray voltage was set at $4.5 \mathrm{kV}$, and capillary temperature was $320^{\circ} \mathrm{C}$. Sheath gas was set to eight arbitrary units and the S-lens Rf level was set to 60. TAGs were separated on a reverse phase column (Luna $3 \mu \mathrm{m} \mathrm{C18} \mathrm{(2)}$ $100 \mathrm{~A}, 150 \times 1.0 \mathrm{~mm}$, (Phenomenex)) at a flow rate of $0.065 \mathrm{ml} / \mathrm{min}$. The column was maintained at $35^{\circ} \mathrm{C}$. The analysis was performed using gradient solvents (A and B) containing $0.1 \% \mathrm{NH}_{4} \mathrm{OH}$. Solvent $\mathrm{A}$ was methanol and solvent $\mathrm{B}$ was propanol. The column was eluted for $2 \mathrm{~min}$ from $0 \% \mathrm{~B}$ to $2 \% \mathrm{~B}$ (linear), from 3 to 6 min with a linear gradient from $2 \%$ solvent B to $3 \%$ solvent B, then isocratically from 3 to 18 min using $3 \%$ solvent B, 18 to $35 \mathrm{~min}$ with a linear gradient from $3 \%$ solvent B to $40 \%$ solvent B, 35 to 60 min using a linear gradient from 40 to $55 \%$ solvent B, then isocratically from 60 to $65 \mathrm{~min}$ at $55 \%$ solvent B then from 65 to 80 min from 55 to $0 \% \mathrm{~B}$ (linear) followed by equilibration from 80 to 90 min at $0 \% \mathrm{~B}$. Standards for TAGs and their oxygenated metabolites were from Avanti Polar Lipids and Cayman Chemicals.

Live Cell Imaging. For the time-lapse imaging, BT-549 cells were seeded in a 6-well plate at 125,000 cells/well. After $24 \mathrm{~h}$, stauroporine $(500 \mathrm{nM})$, $\alpha \mathrm{ESA}(25 \mu \mathrm{M})$ or ML162 (500 nM) were added along with $2 \mathrm{mM}$ HEPES (pH 7.4) and then each well was overlaid with mineral oil and placed in a temperature-controlled imaging chamber $\left(37^{\circ} \mathrm{C}\right)$. Three fields in each well were imaged every $10 \mathrm{~min}$ for a $24 \mathrm{~h}$ period using a Nikon Eclipse TE300 inverted microscope equipped with a Nikon Plan Fluor 10x NA 0.30 objective and a QImaging Retiga EXi camera and using MetaVue software. Movies were assembled using the Fiji implementation of Image $J^{77}$. For photomicrographs, cell images were taken using a Nikon Eclipse TE2000 inverted microscope equipped with Nikon Plan Fluor 10x NA 0.30 objective using Metavue (MetaMorph) or Ocular (QImaging) software.

\section{Detection of lipid peroxidation products. The Click-iT Lipid Peroxidation} Imaging kit (ThermoFisher Scientific) with Alexa Fluor 488 azide was used to detect macromolecules modified with breakdown products from peroxidized lipids according to the recommendations of the manufacturer. Cells were incubated with linoleamide alkyne (LAA) reagent for $16 \mathrm{~h}$ in experiments involving BSO, $4 \mathrm{~h}$ for experiments involving $\alpha E S A$, and $2 \mathrm{~h}$ in experiments using tung oil-conditioned medium before being fixed with fixed with $4 \%$ formaldehyde. DAPI was used to visualize DNA. Fluorescent micrographs were captured with a Nikon Eclipse TE2000 inverted microscope with a Nikon Plan Apo $\times 60$ A/1.40 Oil using Metavue (MetaMorph) or Ocular (QImaging) software except in the experiment using tung oil-conditioned medium where images used for quantification were captured at $\times 20$ magnification using a Zeiss Axio Observer inverted microscope and Zen Blue Software (Zeiss). The fluorescence signal intensity of individual cells was quantified using Image $(\mathrm{NIH})$.

Small interfering RNA. For siRNA knockdown experiments, cells were transfected (DharmaFECT 1, Horizon Discovery) with ON_TARGETplus SMART pools (Horizon Discovery) targeting individual ACSL family members, GPX4 (Catalog ID: L-011676-00), DGAT1 (Catalog ID: L-003922-00), DGAT2 (Catalog ID: L009333-00), ATGL (Catalog ID: L-009003-01) or a non-targeting siRNA pool (Pool \#1, Catalog ID: D-001206-13, Horizon Discovery). For the GPX4 siRNA experiment presented in Fig. 4e, a negative control targeting firefly luciferase was used (Luciferase GL2 Duplex, Catalog ID: D-001100-01, Horizon Discovery). For the ATGL siRNA experiments presented in Supplementary Fig. 6c-g, ON-

TARGETplus a non-targeting control pool was used (Catalog ID: D-001810-10, Horizon Discovery). Efficiency of mRNA depletion was assessed $72 \mathrm{~h}$ posttransfection using qPCR or western blot in the case of ACSL1 (Cell Signaling, Antibody \#4047, used 1:1000), GPX4 (Abcam, [EPNCIR144], ab125066, used 1:1000), DGAT1 (Abcam, [EPR13430-4] - N-terminal ab181180, used 1:5000), DGAT2 (ThermoFisher Scientific, PA5-103785, used 1:1000), and ATGL (Cell Signaling, Antibody \#2138, used 1:1000). $\beta$-actin (Abcam, ab8227, used 1:5000) or GAPDH (Santa Cruz Biotechnology, sc-32233, used 1:5000) were used as loading controls. Secondary antibodies used were goat-anti-mouse-horseradish peroxidase (ThermoFisher Scientific, \#31430, used 1:3000) and goat-anti-rabbit horseradish peroxidase (ThermoFisher Scientific, \#31460, used 1:3000). In experiments to determine suppression of cell toxicity resulting from aESA or ML162 by silencing expression of individual ACSL genes, doses of aESA or ML162 were selected such that there was $5-15 \%$ remaining viability in cells transfected with non-targeting siRNA following $24 \mathrm{~h}$ of treatment

Lentivirus production. Lentiviruses for transductions were packaged and collected from Lenti-X 293 T cells (Takara). The mammalian expression vector along with the lentivirus packaging vector (psPAX2) and envelope vector (pMD2.G) were cotransfected into Lenti-X $293 \mathrm{~T}$ using X-tremeGENE HP DNA transfection reagent (Roche). Lentiviruses were collected at 48,72 , and $96 \mathrm{~h}$ post-transfection and filtered using a $0.22 \mu \mathrm{m}$ membrane. Target cells were incubated with virus-containing 
medium and $2 \mu \mathrm{g} / \mathrm{mL}$ polybrene (Sigma Aldrich) for $24 \mathrm{~h}$, and then allowed recovery for $24 \mathrm{~h}$ prior to selection for transductants.

\section{Stable cell line construction, CRISPR gene editing and gene over-expression.} CRISPR/Cas9 technology was used to generate the ACSL1-deficient lines. 20-bp guide RNA sequences were designed using the CRISPR Design Tool (http://crispr. mit.edu/), and expressed along with Cas9 using the plentiCRISPRv2 vector, which was a gift from Feng Zhang (Addgene plasmid \# 52961; http://www.addgene.org/ search/catalog/plasmids/?q=52961 ${ }^{78}$. Guide RNA sequences are as follows: Nontargeting, scrambled (5'-GCTAAGATCTCGACAACACT-3'), ACSL1 (5'- GTTTC CGAGAGCCTAAACAA- $3^{\prime}$ ). A second independent ACSL1-targeting guide was used to generate the line used in Supplementary Fig. 4g, h (5'- CAAGAGCCATC GCTTCAGCG-3'). After transduction with the relevant guide-containing plentiCRISPRv2, and selection for cells resistant to puromycin (Invivogen, $2 \mu \mathrm{g} / \mathrm{mL}$ ), single cell ACSL1-deficient clones were isolated by plating cells in 96-well plates at a concentration which 1 cell per 3 wells would be expected. Clonal populations were grown in RPMI-1640 containing 20\% FBS without puromycin. Disruption of ACSL1 was determined by western blot. Genomic sequencing confirmed distinct mutations.

Proteins were transgenically over-expressed using the pLX304 vector (Addgene plasmid \#25890; http://www.addgene.org/search/catalog/plasmids/?q=25890). pLX304 was a gift from David Root ${ }^{79}$. For expression of GPX4, a construct containing GPX4 cDNA (GenBank accession BC011836; CCSB Human ORFeome Clone Id 10338, Horizon Discovery), a V5 tag, and the 3'UTR of GPX4 (GenBank accession BC011836) was generated using overlap extension PCR. The primers $\left(5^{\prime}\right.$ to $3^{\prime}$ ) used were as follows: GPX4 ORF AttB1 F (GGGGACAAGTTTGTACAAA AAAGCAGGCTCAATGAGCCTCGGCCGCCTTTGC), GPX4 ORF OL R (ccacttt gtacaagaaagttgggtAGAAATAGTGGGGCAGGTCC), and GPX4 ORF AttB1 R (GGGGACCACTTTGTACAAGAAAGCTGGGTTAATTTGTCTGTTTATTCCC ACAAGG). ACSL1 cDNA was PCR amplified from the MGC Fully Sequenced Human ACSL1 cDNA construct (GenBank accession BC050073; Clone Id 5267238, Horizon Discovery). The primers ( $5^{\prime}$ to $3^{\prime}$ ) used were as follows: ACSL1 F AttB1 (GGGGACAAGTTTGTACAAAAAAGCAGGCTCAATGCAAGCCCATGAGCT GTTC) and ACSL1 R AttB2 (GGGGACCACTTTGTACAAGAAAGCTGGGTT GTAAACCTTGATAGTGGAATAGAGG). To generate the ACSL1 CRISPRresistant mutant, silent mutations in each of the codons (seven total) in the ACSL1 open reading frame recognized by the guide RNA were made using overlap extension PCR. PCR products were subcloned (BP reaction, ThermoFisher Scientific) into pDONR/Zeo (ThermoFisher Scientific) before Gateway cloning into the pLX304 lentiviral expression vector (LR reaction, ThermoFisher Scientific). In addition to the primers used to amplify the CDNA, the following primers $\left(5^{\prime}\right.$ to $\left.3^{\prime}\right)$ were used: ACSL 1 OL Primer 3 F (CATGCTTGGGTTCCCGCAAGCCAGACCAA CCCTATGAATG) and ACSL1 OL Primer 2 R (GAATACAGGTGTCAAATAATG GCCCATGCTTGGGTTCCCGCAAGC). Following lentiviral transduction, blasticidin was used to select for cells containing the pLX304 expression vector (Invivogen, $8 \mu \mathrm{g} / \mathrm{mL}$ for BT-549 and $20 \mu \mathrm{g} / \mathrm{mL}$ MDA-MB-231). Proteins expressed from pLX304 were detected using an anti-V5 antibody (Cell Signaling, (D3H8Q) Rabbit mAb \#13202, used 1:2000). eGFP was used as the negative control (cloned from Addgene plasmid \#25899; http://www.addgene.org/search/catalog/plasmids/? $\mathrm{q}=25899)^{79}$.

Quantitative PCR. Total RNA was isolated using an RNeasy kit (Qiagen) and tested for quality on a Bioanalyzer (Agilent Technologies). RNA concentrations were determined with a NanoDrop spectrophotometer (ThermoFisher Scientific). RNA was reverse transcribed using Moloney murine leukemia virus reverse transcriptase (Ambion- ThermoFisher Scientific) and a mixture of anchored oligo-dT and random decamers (Integrated DNA Technologies). Two reverse-transcription reactions were performed for each sample using either 100 or $25 \mathrm{ng}$ of input RNA. Aliquots of the cDNA were used to measure the expression levels of the genes with the primers, and Power SYBR Green master mix (Applied Biosystems, ThermoFisher Scientific) on a 7900 HT sequence detection system (Applied Biosystems, ThermoFisher Scientific). Cycling conditions were $95^{\circ} \mathrm{C}, 15 \mathrm{~min}$, followed by 40 (two-step) cycles $\left(95^{\circ} \mathrm{C}, 15 \mathrm{~s} ; 60^{\circ} \mathrm{C}, 60 \mathrm{~s}\right)$. Ct (cycle threshold) values were converted to quantities (in arbitrary units) using a standard curve (four points, fourfold dilutions) established with a calibrator sample. The primers $\left(5^{\prime}\right.$ to $\left.3^{\prime}\right)$ used were as follows: ACSL1 (GACATTGGAAAATGGTTACCAAATG, GGCTCACT TCGCATGTAGATA), ACSL3 (CGAAGCTGCTATTTCAGCAAG, CTGTCACC AGACCAGTTTCA), ACSL4 (TCTTCTCCGCTTACACTCTCT, CTTATAAATT CTATCCATGATTTCCGGA), ACSL5 (GGAGAATACATTGCACCAGAGA, AC TCCTACTAAGGATGACCGT), and GPX4 (ACGTCAAATTCGATATGTTCA GC, AAGTTCCACTTGATGGCATTTC). 36B4 was used as the normalizer (CCC ATTCTATCATCAACGGGTACAA, CAGCAAGTGGGAAGGTGTAATCC).

RNA-seq. Total RNA was isolated using the RNeasy kit (Qiagen) from three independent replicates of MDA-MB-231 cells incubated for $5 \mathrm{~h}$ with ML162 $(125 \mathrm{nM})$ or $\mathrm{aESA}(100 \mu \mathrm{M})$ and two replicates from the control incubated with the vehicle for aESA (methanol). Three biological replicate tung-oil or control MDAMB-231 xenograft tumor samples were homogenized in ice-cold PBS using a dounce homogenizer and total RNA was isolated using the RNeasy kit (Qiagen).
The stranded mRNA-seq library was generated from $1000 \mathrm{ng}$ of total RNA from each sample using Truseq stranded mRNA library kit (Illumina) according to the product instructions. In short, mRNAs were enriched twice via poly-T-based RNA purification beads, and subjected to fragmentation at $94^{\circ} \mathrm{C}$ for $8 \mathrm{~min}$ via the divalent cation method. The first strand cDNA was synthesized by SuperscriptII (ThermoFisher Scientific) and random primers at $42^{\circ} \mathrm{C}$ for $15 \mathrm{mins}$, followed by second strand synthesis at $16^{\circ} \mathrm{C}$ for $1 \mathrm{~h}$. During second strand synthesis, the dUTP was used to replace dTTP, thereby the second strand was quenched during amplification. A single ' $\mathrm{A}$ ' nucleotide is added to the 3 ' ends of the blunt fragments at $37^{\circ} \mathrm{C}$ for $30 \mathrm{~min}$. Adapters with illuminaP5, P7 sequences as well as indices were ligated to the cDNA fragment at $30^{\circ} \mathrm{C}$ for $10 \mathrm{~min}$. After Ampure bead (BD) purification (Beckman Coulter), a 15-cycle PCR reaction was used to enrich the fragments. PCR was set at $98^{\circ} \mathrm{C}$ for $10 \mathrm{~s}, 60^{\circ} \mathrm{C}$ for $30 \mathrm{~s}$, and extended at $72{ }^{\circ} \mathrm{C}$ for $30 \mathrm{~s}$. Libraries were again purified using AmPure beads, checked for quality check with a Bioanalyzer (Agilent Technologies) and quantified with Qubit (Invitrogen). Sample libraries were subsequently pooled and loaded to the HiSeq2500 and sequenced using a Hiseq rapid SRcluster kit and HiSeq rapid SBS kit (Illumina). Single $50 \mathrm{bp}$ were generated for the bioinformatic analysis. Raw sequence reads were aligned to the human genome (hg38) using the Tophat algorithm ${ }^{80}$ and Cufflinks algorithm ${ }^{81}$ was implemented to assemble transcripts and estimate their abundance. Cuffdiff ${ }^{82}$ was used to statistically assess expression changes in quantified genes in different conditions.

Cell viability assays and reagents. Cells were seeded in 96-well plates (Corning 3917, 3125-6250 cells per well) and treated with compounds $24 \mathrm{~h}$ after plating. Compounds were purchased from Cayman Chemical with the exception of stearic acid, oleic acid, adrenic acid, linolenic acid, linoleic acid, docosahexaenoic acid, conjugated linoleic acid (16413), bovinic acid, arachidonic acid, Z-VAD-FML, and $(+)$ - $\alpha$-tocopherol (T3634), which were purchased from Sigma Aldrich. RSL3 was purchased from Selleckchem, catalpic acid, $\alpha$-calendic acid, $\beta$-calendic acid, and $\beta$ ESA were obtained from Larodan Fine Chemicals, and punicic acid was purchased from Matreya LLC. Initial aliquots of ferrostatin-1, deferoxamine, and ML162 were generous gifts of the Dixon Lab (Stanford) and additional quantities were purchased from Cayman Chemical. The inhibitor of $\mathrm{FSP}^{9}{ }^{9}$ was purchased from ChemBridge Corp. Staurosporine was purchased from LC Sciences. Cell viability was measured using CellTiter-Glo Luminescent Cell Viability Assay (Promega) according to the manufacturer's instructions. Luminescence was measured on an EnSpire Alpha (Perkin Elmer) using the integrated software package. Data were normalized to vehicle-treated or sensitizing agent-alone controls and $\mathrm{IC}_{50}$ curves were produced with GraphPad Prism (Version 8).

Glutathione measurements. Cellular glutathione was quantified using the GSH/ GSSG-Glo kit (Promega) according to the instructions provided by the manufacturer. Drug-treated samples were normalized to parallel cell viability measurements using the CellTiter-Glo assay (Promega).

Quantitation of GPX4 protein levels. MDA-MB-231 were seeded at a density of 170,000 cells/well of a 6 -well plate. After $24 \mathrm{~h}$, Cells were incubated for $4 \mathrm{~h}$ with either vehicle (methanol or DMSO), ML162 (200 nM), RSL3 (200 nM), erastin $(1 \mu \mathrm{M})$, or $\alpha \mathrm{ESA}(50 \mu \mathrm{M})$. Following treatments, cells were typsinized, pelleted, lysed with RIPA buffer. The western blot was performed using a GPX4 antibody (Abcam, [EPNCIR144], ab125066) (1:1000), $\beta$-actin antibody (Abcam, ab8227) (1:5000) for loading control, and goat anti-rabbit $\operatorname{IgG}(\mathrm{H}+\mathrm{L})$ secondary antibody, HRP (ThermoFisher Scientific, 31460) (1:3000). ImageJ (NIH) was used for the densitometric quantification.

Preparation of tung oil-conditioned medium. Tung oil (Sigma, 440337)-conditioned medium was prepared by combining tung oil and RPMI-1640 complete medium in a 1:2 ratio and emulsifying the components by rotating for $45 \mathrm{~min}$ at room temperature. The emulsion was then centrifuged at $2000 \times \mathrm{g}$ for $10 \mathrm{~min}$ at room temperature. The oil phase was removed and discarded. The aqueous phase was filtered and frozen until further use.

Mouse xenografts. This study was reviewed and approved by the Fox Chase Cancer Center Institutional Animal Care and Use Committee (Protocol \#16-10) and complies with ethical regulations for animal research. Mice were housed in a dedicated laboratory animal facility with 12 -h light:dark cycle, at $70 \mathrm{~F}+/-2$ degrees, and $40-70 \%$ relative humidity. Orthotopic xenografts were generated by implanting 2.5 million MDA-MB-231 cells in $100 \mu \mathrm{L}$ phosphate-buffered saline (PBS) mixed with $100 \mu \mathrm{L}$ growth factor-reduced Matrigel (Corning) bilaterally into the fourth inguinal fat pad of four- to six-week-old female NOD.CgPrkdscid Il2rgtm ${ }^{t m}$ Wjl $/$ SzJ (NSG) mice. After 14 days, when tumors were roughly $50 \mathrm{~mm}^{3}$, animals were randomized into treatment groups. Mice were treated on weekdays with either safflower oil (Whole Foods 365) and Tung oil (Sigma-Aldrich 440337) at a dose of $100 \mu \mathrm{L}$ administered by oral gavage. L-BSO (Sigma) was administered via the drinking water $(20 \mathrm{mM})$ ad libitum, as previously reported 23,83 
Tissue preparation, histology, and immunohistochemistry. Tumor sections and lung sections were fixed in $10 \%$ formalin for $24-48 \mathrm{~h}$, dehydrated, and embedded in paraffin. Immunohistochemical staining was performed by standard methods. To quantify lung metastases, lungs from three mice per treatment group were fixed, sectioned and hematoxylin and eosin stained. Ten equally sized regions ( 2 from each lobe) were randomly selected and scored manually for areas occupied by cancerous tissue. Micrographs were captured with a Nikon DS-Fil camera (Melville, NY, USA) on a Nikon Eclipse 50i microscope and quantified using a ScanScope CS5 slide scanner (Aperio)

\section{Data availability}

All data generated or analyzed during this study are included in this published article (and its supplementary information files) or are available from the corresponding author on reasonable request. Lipidomic data is available at the NIH Common Fund's National Metabolomics Data Repository (NMDR) website, the Metabolomics Workbench, https://www.metabolomicsworkbench.org where it has been assigned Project ID PR000997. The data can be accessed directly via its Project https://doi.org/ 10.21228/M8TH75 [https://www.metabolomicsworkbench.org/data/DRCCMetadata. php? Mode $=$ Project $\&$ ProjectID $=$ PR000997]. RNAseq data is available from the National Center for Biotechnology GEO database (GSE162069). Source data are provided with this paper.

Received: 31 July 2020; Accepted: 11 March 2021;

Published online: 14 April 2021

\section{References}

1. Cao, J. Y. \& Dixon, S. J. Mechanisms of ferroptosis. Cell Mol. Life Sci. 73, 2195-2209 (2016).

2. Xie, Y. et al. Ferroptosis: process and function. Cell Death Differ. 23, 369-379 (2016).

3. Yang, W. S. \& Stockwell, B. R. Ferroptosis: death by lipid peroxidation. Trends Cell Biol. 26, 165-176 (2016)

4. Stockwell, B. R. et al. Ferroptosis: a regulated cell death nexus linking metabolism, redox biology, and disease. Cell 171, 273-285 (2017)

5. Friedmann-Angeli, J. P., Miyamoto, S. \& Schulze, A. Ferroptosis: the greasy side of cell death. Chem. Res. Toxicol. 32, 362-369 (2019).

6. Conrad, M. et al. Regulation of lipid peroxidation and ferroptosis in diverse species. Genes Dev. 32, 602-619 (2018).

7. Hassannia, B., Vandenabeele, P. \& Vanden Berghe, T. Targeting ferroptosis to iron out cancer. Cancer Cell 35, 830-849 (2019).

8. Conrad, M. \& Friedmann Angeli, J. P. Glutathione peroxidase $4(\mathrm{Gpx} 4)$ and ferroptosis: what's so special about it? Mol. Cell Oncol. 2, e995047 (2015).

9. Doll, S. et al. FSP1 is a glutathione-independent ferroptosis suppressor. Nature 575, 693-698 (2019).

10. Bersuker, K. et al. The CoQ oxidoreductase FSP1 acts parallel to GPX4 to inhibit ferroptosis. Nature 575, 688-692 (2019)

11. Gaschler, M. M. et al. FINO2 initiates ferroptosis through GPX4 inactivation and iron oxidation. Nat. Chem. Biol. 14, 507-515 (2018).

12. Conrad, M. \& Pratt, D. A. The chemical basis of ferroptosis. Nat. Chem. Biol. 15, 1137-1147 (2019).

13. Yang, W. S. et al. Peroxidation of polyunsaturated fatty acids by lipoxygenases drives ferroptosis. Proc. Natl Acad. Sci. USA 113, E4966-E4975 (2016).

14. Wenzel, S. E. et al. PEBP1 wardens ferroptosis by enabling lipoxygenase generation of lipid death signals. Cell 171, 628-641 e26 (2017).

15. Zou, Y. et al. Cytochrome P450 oxidoreductase contributes to phospholipid peroxidation in ferroptosis. Nat. Chem. Biol. 16, 302-309 (2020).

16. Agmon, E., Solon, J., Bassereau, P. \& Stockwell, B. R. Modeling the effects of lipid peroxidation during ferroptosis on membrane properties. Sci. Rep. $\mathbf{8}$, 5155 (2018).

17. Kagan, V. E. et al. Oxidized arachidonic and adrenic PEs navigate cells to ferroptosis. Nat. Chem. Biol. 18, 81-90 (2017).

18. Seiler, A. et al. Glutathione peroxidase 4 senses and translates oxidative stress into 12/15-lipoxygenase dependent- and AIF-mediated cell death. Cell Metab. 8, 237-248 (2008).

19. Zou, Y. et al. A GPX4-dependent cancer cell state underlies the clear-cell morphology and confers sensitivity to ferroptosis. Nat. Commun. 10, 1617 (2019).

20. Magtanong, L. et al. Exogenous monounsaturated fatty acids promote a ferroptosis-resistant cell state. Cell Chem. Biol. 26, 420-432 e9 (2019).

21. Tesfay, L. et al. Stearoyl-CoA desaturase 1 protects ovarian cancer cells from ferroptotic cell death. Cancer Res. 79, 5355-5366 (2019).

22. Kamphorst, J. J. et al. Hypoxic and Ras-transformed cells support growth by scavenging unsaturated fatty acids from lysophospholipids. Proc. Natl Acad. Sci. USA 110, 8882-8887 (2013).
23. Beatty, A. et al. Metabolite profiling reveals the glutathione biosynthetic pathway as a therapeutic target in triple-negative breast cancer. Mol. Cancer Ther. 17, 264-275 (2018).

24. Dixon, S. J. et al. Ferroptosis: an iron-dependent form of nonapoptotic cell death. Cell 149, 1060-1072 (2012).

25. Weiwer, M. et al. Development of small-molecule probes that selectively kill cells induced to express mutant RAS. Bioorg. Med. Chem. Lett. 22, 1822-1826 (2012).

26. Cui, P. et al. Tung Tree (Vernicia fordii, Hemsl.) genome and transcriptome sequencing reveals co-ordinate up-regulation of fatty acid beta-oxidation and triacylglycerol biosynthesis pathways during eleostearic acid accumulation in seeds. Plant Cell Physiol. 59, 1990-2003 (2018).

27. Grossmann, M. E. et al. Eleostearic Acid inhibits breast cancer proliferation by means of an oxidation-dependent mechanism. Cancer Prev. Res. 2, 879-886 (2009).

28. Kobori, M., Ohnishi-Kameyama, M., Akimoto, Y., Yukizaki, C. \& Yoshida, M. Alpha-eleostearic acid and its dihydroxy derivative are major apoptosis-inducing components of bitter gourd. J. Agric Food Chem. 56, 10515-10520 (2008).

29. Zhuo, R. J. et al. Alpha-eleostearic acid inhibits growth and induces apoptosis in breast cancer cells via HER2/HER3 signaling. Mol. Med. Rep. 9, 993-998 (2014).

30. Obitsu, S., Sakata, K., Teshima, R. \& Kondo, K. Eleostearic acid induces RIP1mediated atypical apoptosis in a kinase-independent manner via ERK phosphorylation, ROS generation and mitochondrial dysfunction. Cell Death Dis. 4, e674 (2013).

31. Chou, Y. C., Su, H. M., Lai, T. W., Chyuan, J. H. \& Chao, P. M. cis-9, trans-11, trans-13-Conjugated linolenic acid induces apoptosis and sustained ERK phosphorylation in 3T3-L1 preadipocytes. Nutrition 28, 803-811 (2012).

32. Zhang, T. et al. Growth inhibition and apoptotic effect of alpha-eleostearic acid on human breast cancer cells. J. Nat. Med. 66, 77-84 (2012).

33. Yasui, Y. et al. Bitter gourd seed fatty acid rich in $9 c, 11 t, 13 t-c o n j u g a t e d$ linolenic acid induces apoptosis and up-regulates the GADD45, p53 and PPARgamma in human colon cancer Caco-2 cells. Prostaglandins Leukot. Ess. Fat. Acids 73, 113-119 (2005).

34. Yu, C. et al. High-throughput identification of genotype-specific cancer vulnerabilities in mixtures of barcoded tumor cell lines. Nat. Biotechnol. 34 419-423 (2016).

35. Lubbock, A. L. R., Harris, L. A., Quaranta, V., Tyson, D. R. \& Lopez, C. F. Visualization and analysis of high-throughput in vitro dose-response datasets with Thunor. bioRxiv (2019).

36. Grevengoed, T. J., Klett, E. L. \& Coleman, R. A. Acyl-CoA metabolism and partitioning. Annu Rev. Nutr. 34, 1-30 (2014).

37. Dixon, S. J. et al. Human haploid cell genetics reveals roles for lipid metabolism genes in nonapoptotic cell death. ACS Chem. Biol. 10, 1604-1609 (2015).

38. Doll, S. et al. ACSL4 dictates ferroptosis sensitivity by shaping cellular lipid composition. Nat. Chem. Biol. 13, 91-98 (2017).

39. Yuan, H., Li, X., Zhang, X., Kang, R. \& Tang, D. Identification of ACSL4 as a biomarker and contributor of ferroptosis. Biochem Biophys. Res. Commun. 478, 1338-1343 (2016).

40. Wang, W. et al. Tissue-specific ablation of ACSL4 results in disturbed steroidogenesis. Endocrinology 160, 2517-2528 (2019).

41. Li, L. O. et al. Overexpression of rat long chain acyl-coa synthetase 1 alters fatty acid metabolism in rat primary hepatocytes. J. Biol. Chem. 281 37246-37255 (2006).

42. Conlon, M. et al. A compendium of kinetic cell death modulatory profiles identifies ferroptosis regulators. bioRxiv (2019).

43. Do, Q. et al. Development and application of a peroxyl radical clock approach for measuring both hydrogen-atom transfer and peroxyl radical addition rate constants. J. Org. Chem. 86, 153-168 (2020).

44. Garcia-Martinez, M. C., Marquez-Ruiz, G., Fontecha, J. \& Gordon, M. H. Volatile oxidation compouds in a conjugated linoleic acid-rich oil. Food Chem. 113, 926-931 (2009).

45. Kuge, Y., Kanda, A. \& Hara, S. Pursuit of oxidation behavior for conjugated polyenoyl glycerols and establishment of their novel oxidation prevention method. J. Oleo Sci. 58, 295-301 (2009).

46. Luna, P., de la Fuente, M. A., Salvador, D. \& Marquez-Ruiz, G. Differences in oxidation kinetics between conjugated and non-conjugated methyl linoleate. Lipids 42, 1085-1092 (2007).

47. Suzuki, R., Masayuki, A. \& Miyashita, K. Comparative study of the autoxidation of TAG containing conjugated and nonconjugated C18 PUFA. J. Am. Oil Chem. Soc. 81, 563-569 (2004).

48. Morita, M. \& Tokita, M. Hydroxy radical, hexanal, and decadienal generation by autocatalysts in autoxidation of linoleate alone and with eleostearate. Lipids 43, 589-597 (2008)

49. Sousa, B. C., Pitt, A. R. \& Spickett, C. M. Chemistry and analysis of HNE and other prominent carbonyl-containing lipid oxidation compounds. Free Radic. Biol. Med. 111, 294-308 (2017).

50. Dixon, S. J. et al. Pharmacological inhibition of cystine-glutamate exchange induces endoplasmic reticulum stress and ferroptosis. Elife 3, e02523 (2014). 
51. Hassannia, B. et al. Nano-targeted induction of dual ferroptotic mechanisms eradicates high-risk neuroblastoma. J. Clin. Invest. 128, 3341-3355 (2018).

52. Dhar Dubey, K. K., Sharma, G. \& Kumar, A. Conjugated linolenic acids: implication in cancer. J. Agric Food Chem. 67, 6091-6101 (2019).

53. Tsuzuki, T., Tokuyama, Y., Igarashi, M. \& Miyazawa, T. Tumor growth suppression by alpha-eleostearic acid, a linolenic acid isomer with a conjugated triene system, via lipid peroxidation. Carcinogenesis 25, 1417-1425 (2004).

54. Walther, T. C. \& Farese, R. V. Jr Lipid droplets and cellular lipid metabolism. Annu Rev. Biochem. 81, 687-714 (2012).

55. Bailey, A. P. et al. Antioxidant role for lipid droplets in a stem cell niche of drosophila. Cell 163, 340-353 (2015).

56. Jarc, E. et al. Lipid droplets induced by secreted phospholipase A2 and unsaturated fatty acids protect breast cancer cells from nutrient and lipotoxic stress. Biochim Biophys. Acta Mol. Cell Biol. Lipids 1863, 247-265 (2018).

57. Basu, S., Smedman, A. \& Vessby, B. Conjugated linoleic acid induces lipid peroxidation in humans. FEBS Lett. 468, 33-36 (2000).

58. Raff, M. et al. A diet rich in conjugated linoleic acid and butter increases lipid peroxidation but does not affect atherosclerotic, inflammatory, or diabetic risk markers in healthy young men. J. Nutr. 138, 509-514 (2008).

59. Yamasaki, M. et al. Effect of dietary conjugated linoleic acid on lipid peroxidation and histological change in rat liver tissues. J. Agric Food Chem. 48, 6367-6371 (2000).

60. Yang, L., Cao, Y., Chen, J. N. \& Chen, Z. Y. Oxidative stability of conjugated linolenic acids. J. Agric Food Chem. 57, 4212-4217 (2009).

61. Eom, J. M. et al. Alpha-eleostearic acid induces autophagy-dependent cell death through targeting AKT/mTOR and ERK1/2 signal together with the generation of reactive oxygen species. Biochem. Biophys. Res. Commun. 391, 903-908 (2010).

62. Liu, J. et al. Autophagy-dependent ferroptosis: machinery and regulation. Cell Chem. Biol. 27, 420-435 (2020).

63. Friedmann Angeli, J. P., Krysko, D. V. \& Conrad, M. Ferroptosis at the crossroads of cancer-acquired drug resistance and immune evasion. Nat. Rev. Cancer 19, 405-414 (2019).

64. Viswanathan, V. S. et al. Dependency of a therapy-resistant state of cancer cells on a lipid peroxidase pathway. Nature 547, 453-457 (2017).

65. Kyani, A. et al. Discovery and mechanistic elucidation of a class of protein disulfide isomerase inhibitors for the treatment of glioblastoma. ChemMedChem 13, 164-177 (2018).

66. Buccarelli, M. et al. Inhibition of autophagy increases susceptibility of glioblastoma stem cells to temozolomide by igniting ferroptosis. Cell Death Dis. 9, 841 (2018).

67. Chen, L. et al. Erastin sensitizes glioblastoma cells to temozolomide by restraining $\mathrm{xCT}$ and cystathionine-gamma-lyase function. Oncol. Rep. 33, 1465-1474 (2015).

68. Lu, B. et al. The role of ferroptosis in cancer development and treatment response. Front Pharm. 8, 992 (2017).

69. Hangauer, M. J. et al. Drug-tolerant persister cancer cells are vulnerable to GPX4 inhibition. Nature 551, 247-250 (2017).

70. Feng, H. \& Stockwell, B. R. Unsolved mysteries: How does lipid peroxidation cause ferroptosis? PLoS Biol. 16, e2006203 (2018).

71. Soule, H. D. et al. Isolation and characterization of a spontaneously immortalized human breast epithelial cell line, MCF-10. Cancer Res 50, 6075-6086 (1990).

72. Sampaio, J. L. et al. Membrane lipidome of an epithelial cell line. Proc. Natl Acad. Sci. USA 108, 1903-1907 (2011).

73. Ejsing, C. S. et al. Global analysis of the yeast lipidome by quantitative shotgun mass spectrometry. Proc. Natl Acad. Sci. USA 106, 2136-2141 (2009).

74. Surma, M. A. et al. An automated shotgun lipidomics platform for high throughput, comprehensive, and quantitative analysis of blood plasma intact lipids. Eur. J. Lipid Sci. Technol. 117, 1540-1549 (2015).

75. Herzog, R. et al. A novel informatics concept for high-throughput shotgun lipidomics based on the molecular fragmentation query language. Genome Biol. 12, R8 (2011)

76. Herzog, R. et al. LipidXplorer: a software for consensual cross-platform lipidomics. PLoS ONE 7, e29851 (2012).

77. Schindelin, J. et al. Fiji: an open-source platform for biological-image analysis. Nat. Methods 9, 676-682 (2012).

78. Sanjana, N. E., Shalem, O. \& Zhang, F. Improved vectors and genome-wide libraries for CRISPR screening. Nat. Methods 11, 783-784 (2014).
79. Yang, X. et al. A public genome-scale lentiviral expression library of human ORFs. Nat. Methods 8, 659-661 (2011).

80. Trapnell, C., Pachter, L. \& Salzberg, S. L. TopHat: discovering splice junctions with RNA-Seq. Bioinformatics 25, 1105-1111 (2009).

81. Trapnell, C. et al. Transcript assembly and quantification by RNA-Seq reveals unannotated transcripts and isoform switching during cell differentiation. Nat. Biotechnol. 28, 511-515 (2010).

82. Trapnell, C. et al. Differential analysis of gene regulation at transcript resolution with RNA-seq. Nat. Biotechnol. 31, 46-53 (2013).

83. Watanabe, T. et al. A novel model of continuous depletion of glutathione in mice treated with L-buthionine (S,R)-sulfoximine. J. Toxicol. Sci. 28, 455-469 (2003).

\section{Acknowledgements}

This work was supported in part by the Office of the Assistant Secretary of Defense for Health Affairs through the Breast Cancer Research Program of the CDMRP under Award No. W81XWH-19-1-0481. Additional support was provided by National Institutes of Health Awards GM083025, U19AI068021, HL114453-06, CA165065-06, NS076511, the PA Breast Cancer Coalition, the Fifth District AHEPA Cancer Research Foundation, the Spurlino Family Foundation, the Rita Hollman Foundation, the Eileen Stein Jacoby Fund, and Translational Research and In Vino Vita awards from Fox Chase Cancer Center. Supported in part by Award Number P30 CA006927 from the National Cancer Institute of the National Institutes of Health. We thank Drs. S. Balachandran and J. Karanicolas for comments on the manuscript.

\section{Author contributions}

Experiments and data analysis were carried out by A.B., T.S., Y.Y.T., V.A.T., S.S., E.N., K.M., Y.Z., K.Q.C., Y.T., and J.R.P.. S.D. and M.C. provided reagents. A.S. conducted the PRISM cell profiling study. A.B., H.B., V.E.K., U.R., and J.R.P. supervised the work. A.B. and J.R.P. wrote the manuscript with input from the co-authors.

\section{Competing interests}

Ulrike Rennefahrt is a former employee of Metanomics Health, GmbH. Marcus Conrad is an inventor on patents for some of the compounds described herein, and shareholder of ROSCUE Therapeutics GmbH. The remaining authors declare no competing interests.

\section{Additional information}

Supplementary information The online version contains supplementary material available at https://doi.org/10.1038/s41467-021-22471-y.

Correspondence and requests for materials should be addressed to A.B. or J.R.P.

Peer review information Nature Communications thanks Scott Dixon and the other, anonymous, reviewer(s) for their contribution to the peer review of this work. Peer reviewer reports are available.

Reprints and permission information is available at http://www.nature.com/reprints

Publisher's note Springer Nature remains neutral with regard to jurisdictional claims in published maps and institutional affiliations.

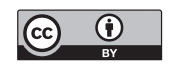

Open Access This article is licensed under a Creative Commons Attribution 4.0 International License, which permits use, sharing, adaptation, distribution and reproduction in any medium or format, as long as you give appropriate credit to the original author(s) and the source, provide a link to the Creative Commons license, and indicate if changes were made. The images or other third party material in this article are included in the article's Creative Commons license, unless indicated otherwise in a credit line to the material. If material is not included in the article's Creative Commons license and your intended use is not permitted by statutory regulation or exceeds the permitted use, you will need to obtain permission directly from the copyright holder. To view a copy of this license, visit http://creativecommons.org/ licenses/by/4.0/.

(C) The Author(s) 2021 